\title{
Microservice security: a systematic literature review
}

\author{
Davide Berardi ${ }^{1}$, Saverio Giallorenzo ${ }^{1,2}$, Jacopo Mauro ${ }^{3}$, Andrea Melis ${ }^{1}$, Fabrizio Montesi ${ }^{3}$, Marco Prandini ${ }^{\text {Corresp. } 1}$ \\ 1 Department of Computer Science and Engineering, University of Bologna, Bologna, Italy \\ 2 INRIA, Sophia Antipolis, France \\ 3 Department of Mathematics and Computer Science, University of Southern Denmark, Odense, Denmark \\ Corresponding Author: Marco Prandini \\ Email address: marco.prandini@unibo.it
}

Microservices is an emerging paradigm for developing distributed systems. With their widespread adoption, more and more work investigated the relation between microservices and security. Alas, the literature on this subject does not form a well-defined corpus: it is spread over many venues and composed of contributions mainly addressing specific scenarios or needs. In this work, we conduct a systematic review of the field, gathering 290 relevant publications-at the time of writing, the largest curated dataset on the topic. We analyse our dataset along two lines: a) quantitatively, through publication metadata, which allows us to chart publication outlets, communities, approaches, and tackled issues; b) qualitatively, through 20 research questions used to provide an aggregated overview of the literature and to spot gaps left open. We summarise our analyses in the conclusion in the form of a call for action to address the main open challenges. 


\title{
Microservice Security: A Systematic Literature Review
}

\author{
Davide Berardi ${ }^{1}$, Saverio Giallorenzo ${ }^{1,2}$, Jacopo Mauro ${ }^{3}$, Andrea Melis ${ }^{1}$, \\ Fabrizio Montesi ${ }^{3}$, and Marco Prandini ${ }^{1}$ \\ ${ }^{1}$ University of Bologna, Italy \\ \{davide.berardi, saverio.giallorenzo2, a.melis, marco.prandini\}@unibo.it \\ ${ }^{2}$ INRIA, France \\ ${ }^{3}$ University of Southern Denmark, Denmark ffmontesi, mauro\}@imada.sdu.dk
}

9 ABSTRACT

Microservices is an emerging paradigm for developing distributed systems that is rapidly approaching widespread adoption. In response to this adoption, an increasing body of work has investigated the relation between microservices and security. Unfortunately, the literature on this subject does not form a well-defined corpus: it is spread over many venues, and it consists of contributions that mainly address specific scenarios or needs. In this work, we conduct a systematic review of the field, gathering 290 relevant publications-at the time of writing, the largest curated dataset on the topic. We analyse our dataset through (i) publication metadata, which allows us to chart publication outlets, communities, approaches, and tackled issues; and (ii) through 20 research questions, which we use to provide an aggregated overview of the literature and to identify gaps left open. Our study leads to a call for action to address the main open challenges.

\section{INTRODUCTION}

Microservices is an emerging development paradigm, where software is built as a composition of multiple services (the "microservices"). Each microservice implements the business logic of a component of the application and is independently executable and deployable. Microservices interact with each other via message-passing APIs (Dragoni et al., 2017).

Over the last 6 years, microservices have become a popular topic and one of the go-to approaches for many cloud computing projects. According to Web of Science, more than 1000 articles about microservices have been published since 2014. The year 2020 accounts for more than 400 of them, which points out that interest in the topic is still rising. Microservices are popular because they bring substantial advantages with respect to scalability in cloud environments and flexibility in the process of software development. By separating application components as independent services, software designers can specialise each component by using a dedicated technology and then integrate all such heterogeneous components via technology-agnostic APIs.

Alas, the advantages of microservices come at a cost: distributed systems are hard to manage, and increasing the number of services of an application gives malicious actors a larger attack surface (Dragoni et al., 2017). Several security concerns that are particularly relevant for microservices have been identified by Chandramouli (2019), and early research has already shown that the application of standard patterns for system reliability needs to take new parameters into consideration - like the locations at which the patterns are deployed (Montesi and Weber, 2018).

The importance of security in microservices creates the need for understanding and analysing the state of the art for securing this kind of architectures. It is particularly important to understand which problems are especially relevant for microservice systems, and how existing techniques can contribute to addressing them. However, there is still a lack of systematic investigations of studies at the intersection of security and microservice architectures.

Here, we aim to fill that gap by presenting a systematic review of the state of the art of microservice security. We followed a structured approach, which led us to select and gather 290 peer-reviewed publications. At the time of this writing, this constitutes the largest curated dataset on the topic. We first perform a quantitative analysis on the metadata of the publications, 
for example, publication outlets and keywords. This provides insight into the communities and key research concepts that currently characterise the field. We then map each publication to a vector of 20 different markers, corresponding to 20 research questions on microservices security that we formulated based on established security techniques and the field of microservices as a whole.

Our research questions focused on threat models, security approaches, infrastructure, and development approach. We perform correlation analysis to show that our questions are well-posed (independence), and also to confirm that some topics correlate positively (e.g., Intrusion Detection and Intrusion Prevention, and Agile Development and DevOps as well). Findings from our analysis include: issues with technology transfer from academia to industry on microservices security; lack of guidelines for adopting security by design in microservices; lack of appropriate threat models; lack of guidelines for addressing the attack surface given by technology heterogeneity; and security issues when migrating systems to microservices. Our data, findings, and discussions form a useful basis for orienting future developments of the field.

In summary, the main contributions of this work are:

- the characterisation of Microservices Security as an early-stage, growing research field in need of systematisation and more mature contributions (Section 5.1.1, Section 5.2.1);

- the identification of the main research communities on the Microservice Security field and the clustering of authors (Section 5.1.2);

- a presentation of the trends of the main security attacks involving microservice architectures, both from the points of view of threat model (Section 5.2.2) and mitigation (Section 5.2.3);

- a report on the current infrastructural security solutions for microservices (Section 5.2.4) as well as the interaction between the main microservices development approaches (such as DevOps and Agile) and security (Section 5.2.5);

- a correlation analysis of the answers to our research questions in papers, which sheds light on relationships among the different aspects of microsrevice security (Section 5.2.7);

- a summary of the main open challenges that emerged from our study, which form a call for action for the community of researchers and practitioners working in the field of microservice security (Section 6).

Structure of the article We start by providing a summary of related work in Section 2. In Section 3 and Section 4 we detail the method we followed to conduct the systematic literature review and the research questions, respectively. We present our results in Section 5 and we conclude in Section 6 with a discussion on the outstanding challenges.

\section{RELATED WORK}

To the best of our knowledge, the published works that are closest to ours are those by Vale et al. (2019) and Almeida et al. (2017). Vale et al. (2019) present a systematic mapping that identifies the security mechanisms used in microservice-based systems. Contrary to our work, which provides a general overview on the state of the art of microservices security, the authors narrow their focus on cataloguing the security technologies and mechanisms adopted by developers of microservice-based systems - e.g., authentication and authorisation - leaving out other subjects related to security, like threat models and development methods. Similarly to Vale et al., Almeida et al. (2017) concentrate on surveying the technologies and standards for security, privacy, and communication used in the area of microservice architectures in the cloud.

Extending our view to articles that, at the time of this writing, are not available as peerreviewed publications, we mention the work by Hannousse and Yahiouche (2020) and Ponce et al. (2021). Hannousse and Yahiouche (2020) present a systematic categorisation of threats on microservice architectures and propose a selection of possible mitigations. Ponce et al. (2021) look at how "security smells" affect microservice-based applications and how to mitigate the effects of such smells through refactoring. As for the proposals by Vale et al. and Almeida 
et al., the difference between our work and Hannousse and Yahiouche (2020) lies on generality: Hannousse and Yahiouche narrow their investigation down to the threats identified in the literature. Similarly, the work of Ponce et al. (2021) focuses on the programming of microservices.

In addition to the related work discussed above, there are quite a few neighbouring surveys with respect to our work that are interesting to discuss: while these studies are not dedicated to the topic of microservice security, they explicitly mention security as an important concern for microservices in different contexts - software engineering, Internet of Things, containerisation, etc. The purpose of reviewing neighbouring related work is twofold:

1. It shows the multifaceted nature of microservice security, giving concrete evidence of the need for an investigation which is both wider and deeper, as we do in this work.

2. It provides a general overview of the challenges and possible uncovered research topics related to security in microservices - which inspired some of the questions presented in Section 3.

Dragoni et al. (2017) present an overview of microservices, including a discussion of the origins of the paradigm, its state of the art, and future challenges. They identify a number of trust and security challenges posed by the paradigm. We mention a few examples. Service reuse, one of the key benefits pushed for in the microservice paradigm, requires adopting secure mechanisms for service authentication and authorisation. The increased granularity and heterogeneity of microservice architectures extends considerably the attack surface of these systems. The sophisticated DevOps infrastructure required to operate microservices effectively is a new attack vector.

Garriga (2017) conducted a preliminary analysis toward a taxonomy of microservices architectures. While not addressing in particular security concerns, Garriga reports that the security subject is not extensively addressed, highlighting how monitoring and microservice communication trust chains should receive particular attention.

Joseph and Chandrasekaran (2019) reviewed approaches proposed in the literature to deal with the various concerns of microservice-based systems. The authors mention the large attack area offered by microservices subject to insider/privilege-escalation attacks and network security issues.

Casale et al. (2016) surveyed the topics of European research projects in the area of software engineering. Regarding microservices security, they highlight four main challenges: increasing the usage of software validation and verification methods; improving the trust and interoperability of services through (self/federated)-certification of outputs based on standards; adopting a securityby-design approach on the whole software lifecycle; and helping developers with addressing discontinuities in the chain of compositionality between services and execution environments - e.g., due to data leakages derived from fragile container-host interactions.

Lichtenthäler et al. (2019) investigate and discuss the challenges of migrating monoliths to microservices. They observe that security should be part of the migration planning phase to begin with, and that developers need models and frameworks to help them elicit, track, and manage the (frequently implicit) assumptions and invariants induced by the migration of the legacy system. These observations are shared with Di Francesco et al. (2017), who suggest that the microservice architectural style has a direct impact on the design of a system and that researchers are still investigating how to leverage its characteristics with respect to system quality and security. Di Francesco et al. note that there exists uncertainty about the realisation of microservices, indicating the need for comprehensive references to help programmers in the multifaceted aspects of microservice development.

Noura et al. (2019) address the open challenges of interoperability in the Internet of Things (IoT), noting how microservices can constitute a solution for the programming of highly distributed IoT networks and provide two decades worth of research and industrial experience to tackle interoperability in heterogeneous systems. Regarding the general security of IoT systems, Noura et al. note the emergence of security issues (e.g., authentication and access control) when system design permits direct access to resource-constrained devices. Reviewing the many solutions and levels at which IoT interoperability can be tackled, Noura et al. note the challenge of 
both maintaining and guaranteeing the same level of security when mediating among different technologies.

Márquez and Astudillo (2019) examine microservice availability tactics to detect, prevent, mitigate, and recover from faults. They highlight how the tactics for the availability of microservices mainly focus on preventing faults, whereas detection, reaction, and recovery are scarcely addressed. Commenting on related challenges, Márquez and Astudillo report a deficit of solutions to support the restoration of normal functionalities after a microservice architecture suffered from some faults.

Ahmed et al. (2019) surveyed robust and flexible service management platforms for IoT systems. Like Noura et al., they identify microservice architectures as the most suitable architectural pattern to handle the heterogeneity of IoT systems and that the foremost challenge in the field is the robust integration of different technologies. Ahmed et al. also report how conventional security solutions and practices are not suitable to handle the expansion, mobility, resource constraints, and new security requirements of the considered systems.

Cerny and Donahoo (2016) investigate service integration from the perspective of separation of concerns and identify problems with conventional service integration design/technologies. They report that the lack of proper cross-cutting concerns in programming technologies make it difficult to capture and guarantee that invariants of a given microservices - specifically, on security - hold when paired with integration components.

Yang et al. (2014) survey how cloud computing systems can help scientific research. In their report, they notice how the (micro)service paradigm is useful to make resources available to collaborating researchers by providing a well-defined interface specifying the operations that can be performed on, or with, a given resource. However, they also report that privacy and trust issues are of particular concern to researchers, especially in fields that are processing sensitive data such as medical research. For this, appropriate provenance metadata is required, both to understand how and by whom the data was created and modified, as well as to understand where it has been potentially exposed to corruption. Similar comments are shared also by Plaza et al. (2018) in the context of healthcare cyber-physical systems. In particular, proper encryption is reported as a key component for (real-time) data acquisition.

Soldani et al. (2018), reviewing the "pains and gains" of microservices in the grey literature, found how security generates pains at design-time. Like Yang et al. (2014), Soldani et al. comment that microservice-based applications should support the consistent determination of the provenance and authenticity of data, noting the paradox of that being in contrast with the heavily-distributed nature of microservice systems. Another (meta)observation by Soldani et al. is how there is a gap between the industrial understanding and state-of-practice on microservices and the state-of-the-art of academic research, one possible reason being that academics have limited access to industrial-scale microservice-based applications.

Di Francesco et al. (2019) identify, classify, and evaluate the state of the art on architecting with microservices from the perspectives of publication trends, the focus of research, and potential for industrial adoption. On security, they report that it is attracting insufficient research. The works by Vural et al. (2017) and Alshuqayran et al. (2016) follow similar modalities and results.

Bélair et al. (2019) surveyed security of containers, a technology frequently paired with microservices. They report how container security is still in an early phase and it faces unsolved challenges. The results presented by Bélair et al. match those by Sultan et al. (2019), who report the presence of a large number of challenges linked to containerisation because OS kernel sharing introduces security issues absent from virtualisation solutions. Sultan et al. also highlight the importance of enhancing vulnerability management, digital investigation, and container alternatives.

Puliafito et al. (2019) present a survey on the employment of fog computing to support IoT devices and (micro)services. In their study, they report how security is the largest crosscutting technical concern within critical IoT systems, which necessitates a common baseline and interoperable standards to address security challenges within both hardware and software. In particular, Puliafito et al. advocate for solutions to provide a full-stack secure chain of trust from devices to fog/cloud components, which has been only preliminary explored (as remote attestation techniques). Trnka et al. (2018) and Puliafito et al. report also the importance of 


\begin{tabular}{|c|c|c|c|c|c|c|}
\hline Publication & Year & Type & Num. & White L. & Grey L. & Sources \\
\hline This work & 2021 & SLR & 290 & $\bullet$ & ○ & $\begin{array}{c}\text { ACM Digital Library } \\
\text { IEEE Xplorer } \\
\text { SpringerLink } \\
\text { Scopus } \\
\text { Science Direct } \\
\text { Wiley } \\
\text { Google Scholar }\end{array}$ \\
\hline Vale et al. & 2019 & SLR & 26 & $\bullet$ & O & $\begin{array}{c}\text { ACM Digital Library } \\
\text { IEEE Xplorer } \\
\text { SpringerLink } \\
\text { Science Direct } \\
\text { Wiley } \\
\text { Google Scholar }\end{array}$ \\
\hline Almeida et al. & 2017 & Survey & N.A. & $\bullet$ & o & N.A. \\
\hline Hannousse and Yahiouche & 2020 & SLR & 46 & $\bullet$ & o & $\begin{array}{l}\text { ACM Digital Library } \\
\text { IEEE Xplorer } \\
\text { SpringerLink } \\
\text { Science Direct } \\
\text { Wiley }\end{array}$ \\
\hline Soldani et al. & 2018 & SLR & 51 & ○ & $\bullet$ & $\begin{array}{c}\text { Google } \\
\text { Bing } \\
\text { Duck Duck Go } \\
\text { Yahoo! } \\
\text { Webopedia } \\
\end{array}$ \\
\hline
\end{tabular}

Table 1. Summary table and comparison with related works. For each row/work in the table, we report: its reference; its publication year; its type (systematic literature review (SLR), survey, etc.); the number of publications it encompasses; whether it analyses white (peer reviewed) literature; whether it analyses grey (blog posts, etc) literature; the sources it used to search its dataset.

addressing the concerns of context-aware security (in IoT systems), especially for authentication and authorisation.

Also Yu et al. (2019) surveyed the literature on microservice-based fog applications to elicit the security risks threatening them. The main threats highlighted include: kernel-level leakage vulnerabilities linked to containerised deployment; man-in-the-middle/insider attacks on datatransmission interception; the need to verify when services become compromised/misbehave; and network-level vulnerabilities on data-routing alteration.

The table 1, shows the differences between these various works, in numerical and boolean terms. As clearly evincible, our work expands the previous works by adding a conspicuous amount of analysed publications; using white literature at its roots and following the trend and methods of the main Systematic White Literature Reviews.

\section{REVIEW METHOD}

In this section, we describe and motivate the steps we followed to perform our systematic review.

Following the guidelines by Snyder (2019), and as depicted in Fig. 1, we started by searching and retrieving the literature for relevant publications from several data sources by using the same keyword query. We then performed a manual revision process of the automatically selected publications to exclude publications out of the scope of this study and perform snowballing-i.e., recursively adding to the dataset relevant publications cited by the already selected publications. The resulting dataset consists of 290 publications. We analysed these publications to collect statistical and transparent answers to our research questions, which are detailed in Section $4 .^{1}$

\footnotetext{
${ }^{1}$ The list of the publications and their bibliography information is publicly available at https://doi.org/10 5281 /zenodo. 4774894 .
} 


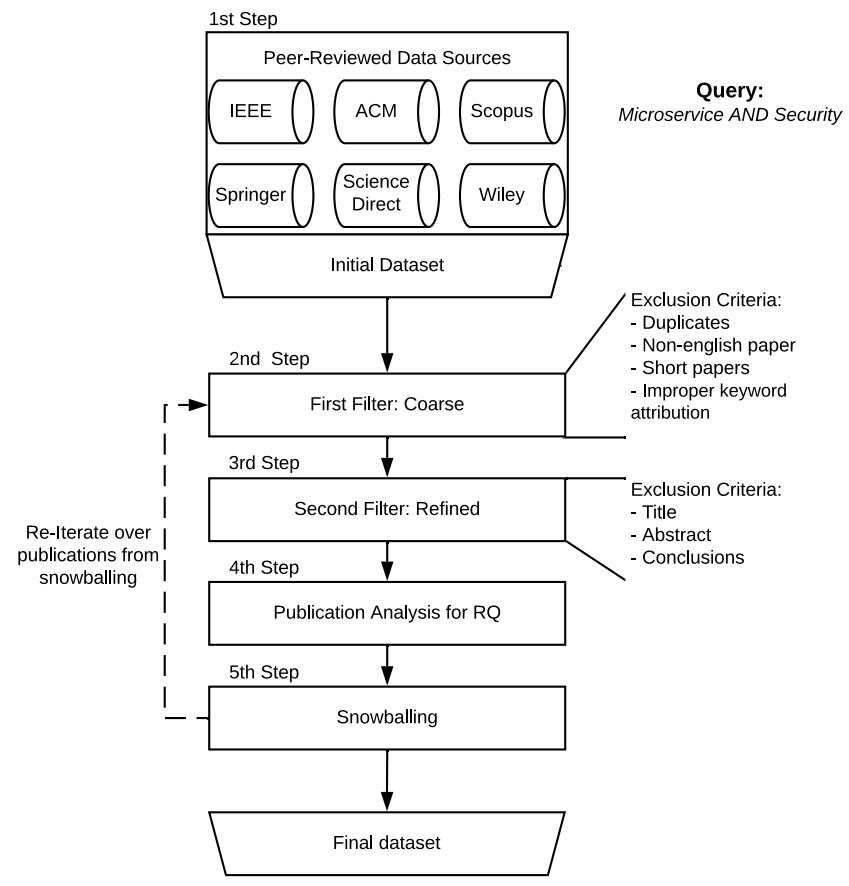

Figure 1. Schema of the method followed to gather the dataset for this review.

\subsection{Selection Query and Collection of Publications}

Security in microservices includes complex and heterogeneous topics, ranging from development to infrastructural concerns. In our choice of a selection query to gather an initial dataset, it was important to pick a sufficiently general query. For this reason, we adopted the query "Microservice AND Security" for our initial search, capturing all the publications containing both terms in any of their title, abstract, or body. ${ }^{2}$

Di Francesco et al. (2019); Plaza et al. (2018); Soldani et al. (2018) reported how publications on the topic of Microservice started in 2014. Taking into account this fact, we limited our research to contributions published since 2014. During the seven years covered by our work, the body of knowledge on this topic has grown significantly. For this reason, we deemed it useful to consider white literature only: in terms of quantity, it represents a very meaningful sample of the research produced during the considered time frame, and in terms of quality, it allowed us to rely on peer review. Thanks to the more uniform organisation of white literature, we are also more confident in the level of consistency of our choice and application of the selection criteria. This is not to say that grey literature is not worth investigating. Blog posts, personal websites, technical reports, white papers, etc., are often the preferred venues for practitioners to share ideas. However, as also pointed out in Soldani (2019), "it is very difficult to uniquely measure the quality of grey literature when conducting a systematic, controllable, and replicable secondary study" and we are not aware of a standard method for the evaluation of grey literature. Analysing the grey literature was beyond the quality goal of this article and we leave it as future work.

Accordingly to this strategy, we collected publications from 6 different publishers, focusing on peer-reviewed publications. We did not, for example, use Google Scholar or arXiv, since they also list resources that are not peer-reviewed. We list the publishers, reporting the respective numbers of publications that matched our query:

\footnotetext{
${ }^{2}$ We performed experiments with potentially more inclusive queries, such as "Microservice AND (Security OR Authorisation)", as well. The tried queries, however, did not extend the search in any useful way since the term "Security" proved to be general enough to cover specialised aspects like authentication, authorisation, and (safe) communication.
} 
- ACM (https://dl.acm.org/), 478 publications;

- IEEE explore (https://ieeexplore.ieee.org/), 181 publications;

- Springer (https://link.springer.com/), 345 publications;

- Scopus (https://www.scopus.com/home.uri), 134 publications;

- Science Direct (https://www.sciencedirect.com/), 358 publications;

- Wiley (https://onlinelibrary.wiley.com/), 208 publications.

This gave us an initial dataset of 1704 publications in total. We collected publications published up to the 31st of December 2020, using the academic subscriptions provided by the affiliations of the authors - the University of Bologna and the University of Southern Denmark. To guarantee the same level of trustworthiness and authenticity, we retrieved the publications only from the official entries avoiding external sources such as the authors' personal websites.

\subsection{Publications Triage}

The publications retrieved from the publishers were processed in three steps to check if they should be excluded according to distinct exclusion criteria. Graphically, in Fig. 1, these steps are labelled as 2nd, 3rd, and 4th Step(s).

In the 2nd Step, we looked at whether the keywords "Microservice" and "Security" were used. We excluded a publication if the keywords appeared only in the bibliography. Moreover, we excluded the publication if it was too short (less than two pages), publications not written in English, and duplicate publications already listed in another publisher source.

In the 3rd Step, we looked at the title, abstract, and conclusion of each publication. Publications that do not treat or discuss topics related to microservices and security were excluded. In this step, we also excluded publications in which the security topic was orthogonal or incidental. In this way, we excluded publications where "microservices and security" was one of the possible application scenarios, but not the main subject of the study. We also excluded cases in which the work tangentially mentioned the satisfaction of some security aspects, without detailing the design/development of the security technologies to accomplish them. For example, we excluded publications focusing on blockchain technologies where the authors incidentally mention authentication and integrity protection as inherent security properties of blockchain-based implementations.

In the 4th Step, we performed an analysis of the publications, answering to the research questions (RQ) detailed in Section 4. No publications were excluded at this step.

At this point, the following publications remained in the dataset (268 in total):

- ACM, 67 publications;

- IEEE explore, 59 publications;

- Springer, 46 publications;

- Scopus, 28 publications;

- Science Direct, 53 publications;

- Wiley, 15 publications.

\subsection{Snowballing}

As the last (5th) step for the systematic literature review, we performed a backward snowballing process (Wohlin, 2014) with the objective of identifying additional relevant references for our study from the works cited by the already selected publications.

All references collected in this way underwent the triage by following the Steps 2, 3, and 4. Each referenced publication accepted for inclusion by these steps was then added to the dataset of selected publications. Snowballing was recursively performed on these newly-added publications until reaching a fixed point; i.e., until no new publications was added to the dataset.

The outcome of repeatedly applying the snowballing process led to the following results: 
- 40 references in the first round, from which we selected 9 publications;

- 22 references in the second round, from which we selected 8 publications;

- 5 references in the third round, from which we selected 5 publications;

- 4 references in the fourth round, where we selected 0 publications.

The 4 cycles of snowballing yielded 22 additional publications that were included in the dataset to reach the final size of 290 publications.

\section{RESEARCH QUESTIONS}

In this section, we detail the research questions that guided our systematic review.

Usually, the research questions for systematic literature reviews are fairly broad and do not amount to more than six. In our case, we chose to adopt more questions (20) but dichotomous (i.e., with yes-or-no answers), to favour precision and objectiveness. To define the questions and seek guidance in categorising the relevant security issues for microservices, we took inspiration from the related work presented Section 2, as well as from the state of the art in standards and methods, namely the NIST Special Publication 800-204 "Security Strategies for Microservicebased Application Systems" (Chandramouli, 2019).

Our questions are collected in four macro groups (Gs), each covering a different concern.

- G1: Threat Model. Questions on threat modelling and how threats are dealt with.

- G2: Security Approach. Questions on the security approach, e.g., whether it is preventive, adaptive, proactive, or reactive.

- G3: Infrastructure. Questions on the infrastructure that microservices run on.

- G4: Development. Questions on the development process.

The questions in each group are reported in the remainder of this section.

\subsection{First group: Threat Model}

Mapping the usage of threat models is important to see gaps when a security violation must be handled, or if known models are outdated and need to be adjusted. The NIST report, for instance, hints at the importance of identifying the threats looming over a microservices architecture (Chandramouli, 2019). The usage of a formal threat model has proven to be extremely useful in the identification of attack types and their strategic countermeasures (Death, 2017).

Several threat models exist in the literature. The most famous one is STRIDE (Kohnfelder and Garg, 1999) named after the Spoofing, Tampering, Repudiation, Information Disclosure, Denial of Service, and Elevation of privilege security threats. Other threat models however exists, such as PASTA (UcedaVelez and Morana, 2015) or OWASP (OWASP Foundation, 2020).

In our review and with this first group of questions, we aimed to understand whether a publication followed a known model, strategy, or guideline. Alternatively, we wanted to know if new security models were proposed.

This group consists of the following questions.

- Q1: Does the publication mention STRIDE, or at least consider all of its aspects?

- Q2: Even without explicitly mentioning STRIDE, does the publication involve at least one of its aspects (Spoofing, Tampering, ...)?

- Q3: If STRIDE aspects or equivalent are considered, does the publication propose/discuss a concrete implementation/solution (either developed by the same author or one taken from the literature)?

- Q4: Does the publication consider or follow another threat model rather than STRIDE without introducing a new one? 
- Q5: Does the publication mention policies, workflows, or guidelines to handle violations?

In particular, with question Q1 and Q3 we looked for the adoption of STRIDE, being the most popular threat model. In the remaining questions, we investigate if the publication defined some threat model - either from the literature or a newly one introduced in that publication — or at least discussed equivalent principles or guidelines without mentioning STRIDE.

\subsection{Second Group: Security Approach}

Many related works cite the usage of preventive measures to secure microservices (Márquez and Astudillo, 2019; Vale et al., 2019; Garriga, 2017; Almeida et al., 2017; Ahmed et al., 2019; Soldani et al., 2018) while some indicate the need for further research in the other directions of proaction, reaction, and adaptation (Vale et al., 2019; Márquez and Astudillo, 2019). With this second block of questions, we wanted to go deeper into the security aspects, considering the specific security approaches, solutions, and also the role that microservices play.

This group consists of the following questions.

- Q6: Does the publication mention Intrusion Detection System (IDS) functionalities?

- Q7: Does the publication mention Intrusion Prevention Systems (IPS) functionalities?

- Q8: Does the publication mention Threat Intelligence?

- Q9: Does the publication mention Exfiltration Leaks?

- Q10: Does the publication address Insider Threats?

- Q11: Are microservices part of the solution?

- Q12: Are privacy and GDPR considered?

\subsection{Third Group: Infrastructure}

The NIST report by Chandramouli (2019) dedicates a large part of its content to infrastructural security solutions for microservices. Similarly, the majority of the mentioned related work in Section 2 presents or at least cites infrastructural solutions for security, acknowledging that the infrastructure of microservice systems is typically complex, encompassing concerns that span from service deployment and service-to-service coordination (discovery, composition, consistency) to the definition of security-specific mechanisms (authorisation, authentication).

In this group of questions, we aimed at finding information on the infrastructure configurations considered in the publication. This group consists of the following questions.

- Q13: Does the publication specify how the proposed architecture is controlled or managed (e.g., in a centralised, decentralised, or hybrid way)?

- Q14: Does the publication mention Infrastructure-as-a-Service?

- Q15: Does the publication mention service discovery?

\subsection{Fourth Group: Development}

Microservices are often associated with software development practices like DevOps and Agile (Balalaie et al., 2016; Vadapalli, 2018) which, in turn, are heavily influenced by the inclusion of security-oriented practices (Casale et al., 2016; Lichtenthäler et al., 2019; Cerny and Donahoo, 2016; Soldani et al., 2018).

In this last set of questions, we aimed at checking the extent to which these practices are used also in the setting of security, for example by verifying whether specific development processes and security standards are considered.

This group consists of the following questions.

- Q16: Does the publication mention DevOps, Continuous Integration, Continuous Deployment, or Continuous Delivery? 


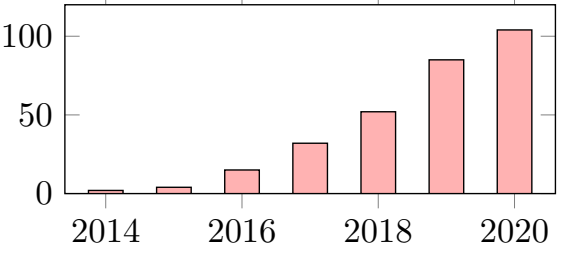

(a) Time distribution.

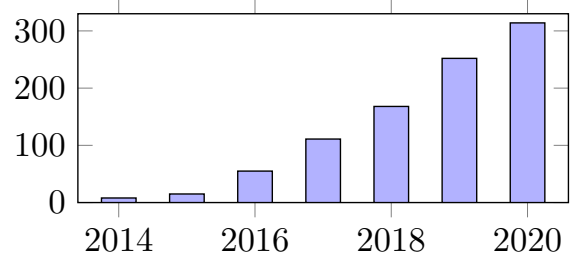

(b) Time distribution, ppm.

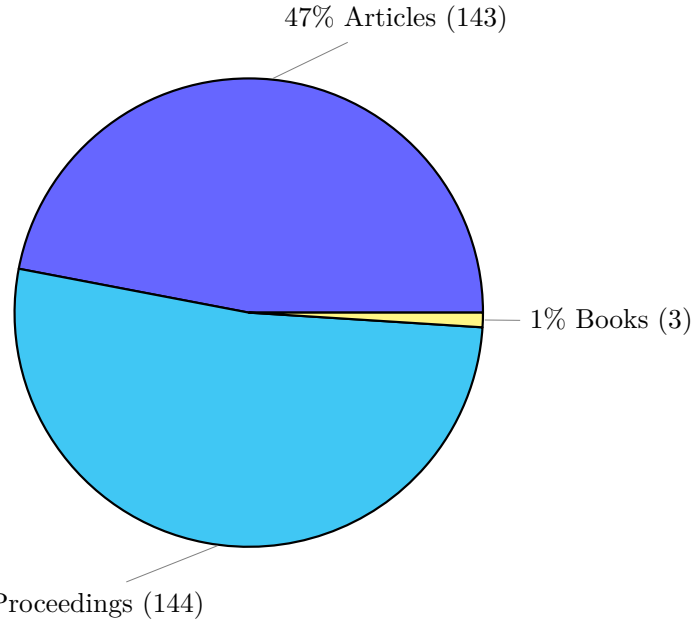

(c) Category

Figure 2. Time and category distribution of publications.

- Q17: Does the publication mention Agile, or how security experts are integrated from a development process point of view?

- Q18: Does the publication mention Domain Driven Development?

- Q19: Does the publication mention Model Driven Development?

- Q20: Does the publication mention certifications, such as $\mathrm{ISO} 27000^{3}$, or technological standards such as X.5094?

\section{REVIEW RESULTS}

In this section, we present the outcome of the literature review. We start by presenting quantitative results obtained from the metadata of the publications in our dataset. This is useful to map the trends over time and the current shape of the field, in terms of the number of contributions, type (proceedings, articles), communities, and keywords (and their relations). Then, we present results derived from the analysis of the types of contributions (theoretical, applicative, etc.) and of the relation between the selected dataset and our research questions (cf. Section 4). This part is aimed at providing a detailed insight on existing research patterns, gaps, and uncovered areas of the field. We close the subsection with a correlation analysis of the questions, providing a quantitative look over the relationships between them. For reference, we also report our dataset in tabular form, where each entry is associated with the positive answers given to our research questions.

\section{Insights}

In the following subsections, we highlight in boxes (like this one) the main insights that emerge from our analysis. Each insight motivates an open challenge, which we write in bold as the heading of the insight. We will use these challenges in Section 6 to structure our discussion about useful future directions for research on microservice security.

\subsection{Metadata analysis}

We start our quantitative analysis of the collected dataset by presenting in Fig. 2(a) the time distribution of the selected publications. As expected, security in microservice systems gained a

\footnotetext{
${ }^{3}$ https://www.iso.org/isoiec-27001-information-security.html

${ }^{4}$ https://tools.ietf.org/html/rfc5280
} 


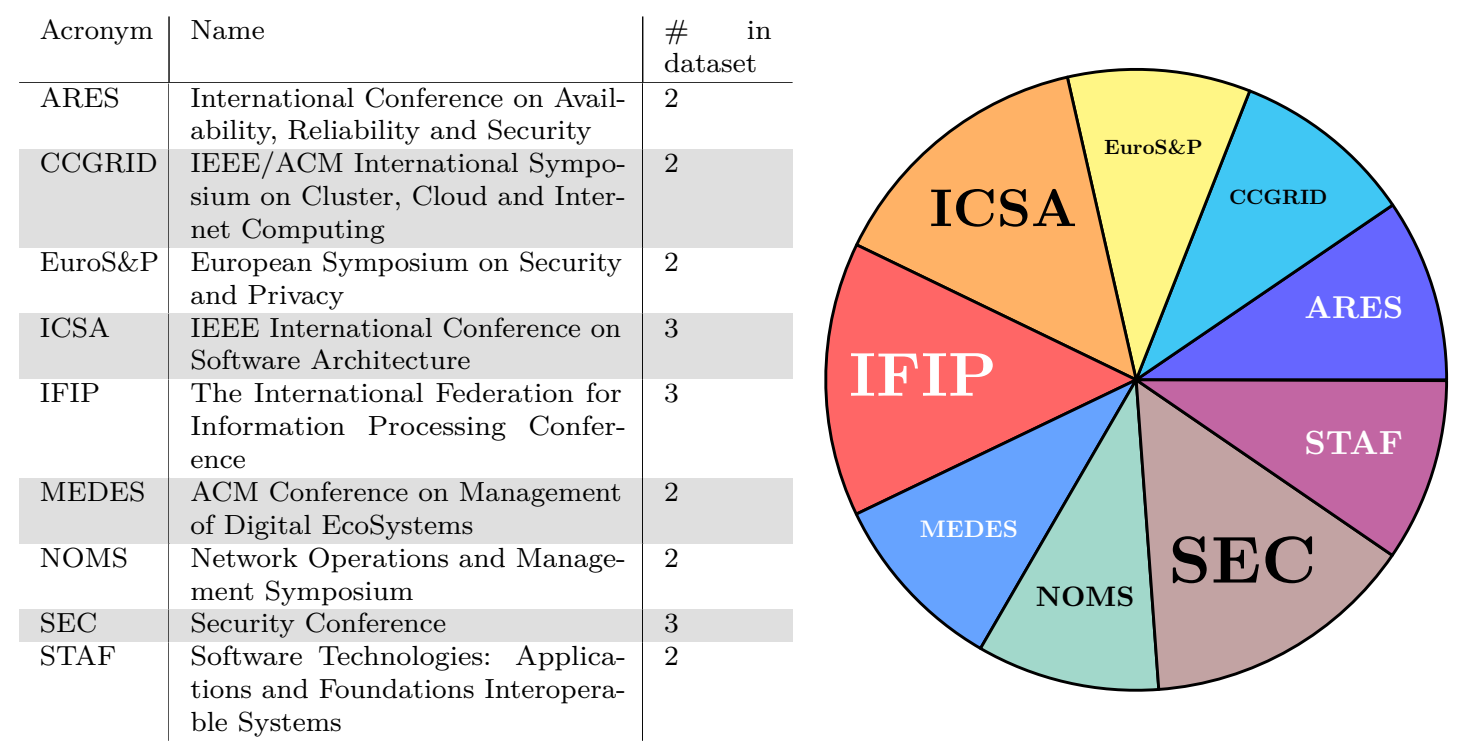

Figure 3. Conferences with the largest number of publications in our dataset.

lot of academic interest in the latest years. This is reflected by the sharp increase in the number of publications since 2014. In Fig. 2(a), we report the number of collected publications per year. As a reference to indicate the degree of growth of the field, we report in Fig. 2(b) the yearly ratio (in parts per million) between the collected publications and the overall number of publications in computer science ${ }^{5}$.

\subsubsection{Publication Outlets}

From the plot in Fig. 2(c) we see that conferences and journal venues are the most common outlets, while books/collections are underrepresented. This last fact indicates the early stage of the field, where established references are still lacking. However, conference proceedings are almost matched by journal articles, marking a maturing trend of results that are solid enough to constitute material for more structured contributions, as those found in peer-reviewed journals.

We now concentrate on the specific conferences and journals where the publications in our dataset have been published. In Figs. 3 and 4, we report this result in two versions: $i$ ) in tabular form, on the left-hand side of Figs. 3 and 4, with the acronym, the full name, and the number of contributions in our dataset of the venues with the most contributions and $i i$ ) on the right-hand side of Figs. 3 and 4, showing the data on the left as a pie chart.

Regarding the distribution of publications over the different categories of venues, we note how the audience of journals and conferences vary. In fact, there is no predominance of securityoriented or even software engineering venues, which could have been the most likely targets. Instead, the analysed publications appear at venues addressing a broad range of topics, from networking to cloud computing, and on open journals such as IEEE Access and ACM Queue. Furthermore, there is no clear preferred venue that dominates the others, but contributors are rather scattered over many neighbouring venues.

We give a twofold interpretation of the phenomenon. On the one hand, this fact can indicate that microservice security is perceived as of cross-disciplinary interest, each contribution seeing it from the lens of its specific area (whether it be software engineering, networks, sensors, cloud computing, etc.). On the other hand, we notice the lack of specific venues dedicated to microservices, and least of all, dedicated to microservice security.

${ }^{5}$ Source: https://dblp.org/statistics/publicationsperyear.html. 


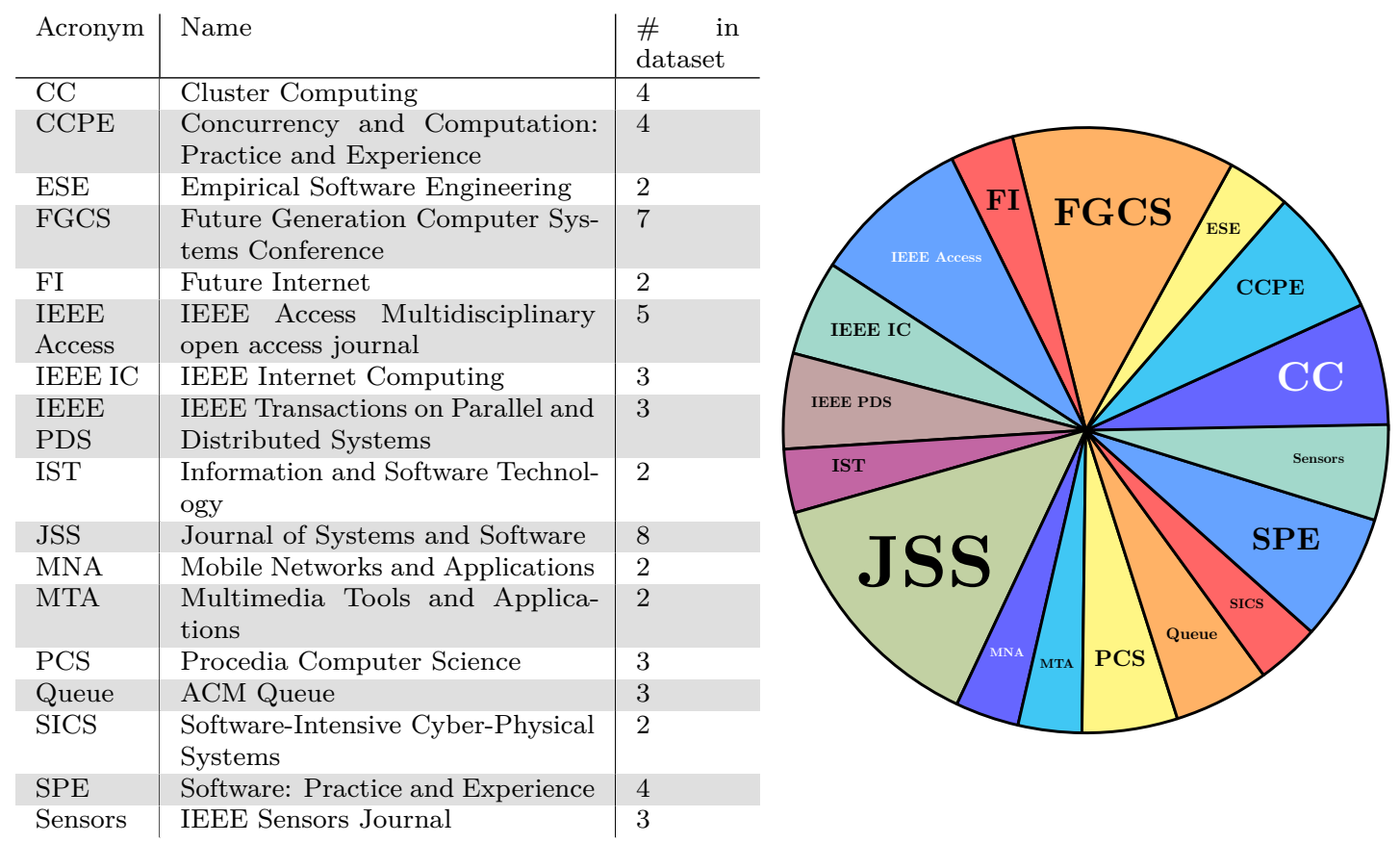

Figure 4. Journals with the largest number of publications in our dataset.

\section{Insights}

Fragmentation of outlets: there are no reference venues for the area of microservice security (neither journals nor conferences). This makes it difficult for researchers and practitioners to keep up with the state of the art, as well as to find dedicated conventions where they can discuss this topic with the rest of the community interested in the area.

\subsubsection{Research Communities}

To add more insight on the communities of the field, we also perform a network analysis to identify and explore the clusters of the most prolific authors and their research collaborations. Specifically, we are interested in analysing the networks of collaboration of "core authors", i.e., prolific authors that, by working with different people, act as a liaison among separated groups of authors.

To find the clusters of core authors in our dataset, we consider all the authors in the dataset and we aggregate them in clusters such that each member of a cluster has at least one contribution published with one of the members of the cluster. Since we are interested in "core authors"-i.e., authors with more than 2 works in the dataset - we remove all those clusters formed around just one work - i.e., where the maximum number of publications published by the most prolific author is one. 


\begin{tabular}{|c|c|c|c|c|c|c|c|}
\hline Cluster & Author & \# pub. & Affiliation & Cluster & Author & \# pub. & Affiliation \\
\hline A & Fetzer Christof & 3 & TU Dresden & $\mathrm{G}$ & Makitalo Niko & 1 & University of Helsinki \\
\hline $\mathrm{A}$ & Brito Andrey & 2 & Universidadede Campina Grande & $\mathrm{H}$ & Jin Yike & 1 & Unknown affiliation \\
\hline A & Kopsell Stefan & 2 & $\begin{array}{l}\text { TU Dresden } \\
\end{array}$ & $\mathrm{H}$ & Yu Dongjin & 1 & Hangzhou Dianzi University \\
\hline A & Pietzuch Peter & 2 & Imperial College London & $\mathrm{H}$ & Zhang Yuqun & 1 & Southern University \\
\hline A & Pasin Marcelo & 2 & University de Neuchâtel & $\mathrm{H}$ & Zheng Xi & 3 & Xi'an Jiaotong University \\
\hline A & Felber Pascal & 2 & University of Neuchâtel & $\mathrm{H}$ & Zhang Chong & 2 & Chong Qing Hospital \\
\hline A & Fonseca Keiko & 1 & Universidade do Paraná & $\mathrm{H}$ & Liu Xiao & 2 & Tsinghua University \\
\hline A & Rosa Marcelo & 1 & University of Melbourne & $\mathrm{H}$ & Li Rui & 2 & Facebook \\
\hline $\mathrm{A}$ & Gomes Luiz & 1 & Arizona State University & $\mathrm{H}$ & Liu Huai & 2 & University of Washington \\
\hline $\mathrm{A}$ & Riella Rodrigo & 1 & Universidade do Paraná & $\mathrm{I}$ & Donahoo Michael J & 2 & Carnegie University \\
\hline A & da Silva MS Leite & 1 & Universidade Campina Grande & I & Cerny Tomas & 6 & Baylor University \\
\hline A & de Oliveira SV Fernando & 1 & Universidade de Campina Grande & I & Sedlisky Filip & 1 & University In Prague \\
\hline A & Kelbert Florian & 1 & Elastic & I & Walker Andrew & 2 & Carnegie University \\
\hline A & Gregor Franz & 1 & TU Dresden & I & Svacina Jan & 2 & Baylor University \\
\hline A & Pires Rafael & 1 & University of Sao Paulo & I & Bushong Vincent & 2 & Baylor University \\
\hline A & Schiavoni Valerio & 1 & University of Neuchâtel & I & Bures Miroslav & 2 & University In Prague \\
\hline A & Mazzeo Giovanni & 2 & MDM-IMM-CNR lab & I & Tisnovsky Pavel & 2 & University In Prague \\
\hline A & Oliver John & 1 & UC Berkeley & I & Frajtak Karel & 2 & University in Prague \\
\hline A & Romano Luigi & 1 & Università della Campania & I & Shin Dongwan & 2 & Korea Institute of Energy Research \\
\hline A & Brenner Stefan & 1 & TU Braunschweig & I & Huang Jun & 2 & Duke University \\
\hline A & Hundt Tobias & 1 & UCL Institute of Child Health & $\mathrm{J}$ & Yarygina Tetiana & 4 & University of Bergen \\
\hline $\mathrm{A}$ & Kapitza Rudiger & 1 & TU Braunschweig & $\mathrm{J}$ & Otterstad Christian & 3 & University of Oslo \\
\hline $\mathrm{B}$ & Artac & 1 & Necmettin Erbakan University & $\mathrm{J}$ & Lysne Olav & 1 & Simula Research Laboratory \\
\hline B & Casale Giuliano & 2 & Imperial College London & $\mathrm{J}$ & Hole Kjell J & 1 & Simula Research Laboratory \\
\hline B & Van Den Heuvel W-J & 2 & Tilburg University & $\mathrm{J}$ & Ytrehus & 1 & University of Tromso \\
\hline $\mathrm{B}$ & van Hoorn Andre & 5 & University of Stuttgart & $\mathrm{J}$ & Aarseth Raymond & 1 & University of Tromso \\
\hline B & Jakovits Pelle & 1 & University of Tartu & $\mathrm{J}$ & Tellnes Jorgen & 1 & University of Bergen \\
\hline B & Leymann Frank & 1 & University of Stuttgart & $\mathrm{J}$ & Bagge Anya Helene & 1 & University of Bergen \\
\hline $\mathrm{B}$ & Long Madeleine & 1 & University of Oslo & K & Cecconi Alessio & 1 & Vienna University \\
\hline $\mathrm{B}$ & Papanikolaou Vicky & 1 & National School of Public Health & $\mathrm{K}$ & Di Ciccio Claudio & 1 & Sapienza University of Rome \\
\hline B & Presenza Domenico & 1 & University of Rome & $\mathrm{K}$ & Dumas Marlon & 1 & University of Tartu \\
\hline $\mathrm{B}$ & Russo Alessandra & 1 & University of Catania & $\mathrm{K}$ & Garcia-Banuelos Luciano & 1 & Tecnologico de Monterrey \\
\hline B & Chesta Cristina & 1 & University of Chester & $\mathrm{K}$ & Lopez-Pintado Orlenys & 1 & University of Tartu \\
\hline B & Di Nitto Elisabetta & 1 & Politecnico di Milano & $\mathrm{K}$ & Lu Qinghua & 3 & universtiy of delaware \\
\hline $\mathrm{B}$ & Gouvas Panagiotis & 2 & University of Athens & K & Mendling Jan & 1 & Humboldt-Universität zu Berlin \\
\hline $\mathrm{B}$ & Stankovski Vlado & 2 & University of Ljubljana & K & Tran An Binh & 1 & CSIRO \\
\hline $\mathrm{B}$ & Symeonidis Andreas & 1 & University of Thessaloniki & $\mathrm{K}$ & Weber Ingo & 3 & TU Berlin \\
\hline $\mathrm{B}$ & Zafeiropoulos Anastasios & 2 & University of Athens & $\mathrm{K}$ & Binh Tran An & 2 & CSIRO \\
\hline $\mathrm{B}$ & Soldani Jacopo & 1 & University of Pisa & $\mathrm{K}$ & O'Connor Hugo & 2 & CSIRO \\
\hline B & Avritzer Alberto & 4 & eSulabSolutions & $\mathrm{K}$ & Rimba Paul & 2 & CSIRO \\
\hline B & Ferme Vincenzo & 3 & Kiratech S.p.A. & $\mathrm{K}$ & Xu Xiwei & 2 & National Institute of Natural Hazards \\
\hline $\mathrm{B}$ & Janes Andrea & 3 & The James Hutton Institute & $\mathrm{K}$ & Staples Mark & 2 & CSIRO \\
\hline $\mathrm{B}$ & Russo Barbara & 3 & Free University of Bozen-Bolzano & K & Zhu Liming & 3 & CSIRO \\
\hline $\mathrm{B}$ & Schulz Henning & 3 & Novatec Consulting GmbH & $\mathrm{K}$ & Jeffery Ross & 2 & Mayo Clinic \\
\hline B & Menasche & 3 & University of Rio de Janeiro & $\mathrm{L}$ & Mirri Silvia & 2 & University of Bologna \\
\hline $\mathrm{B}$ & Rufino Vilc & 3 & UFRJ & $\mathrm{L}$ & Melis Andrea & 4 & University of Bologna \\
\hline B & Trubiani Catia & 1 & Gran Sasso Science Institute & $\mathrm{L}$ & Prandi Catia & 2 & University of Bologna \\
\hline B & Bran Alexander & 1 & University of Exeter & $\mathrm{L}$ & Prandini Marco & 4 & University of Bologna \\
\hline $\mathrm{C}$ & Rocha Carla & 1 & Rutgers University & $\mathrm{L}$ & Salomoni Paola & 2 & University of Bologna \\
\hline $\mathrm{C}$ & Leite Leonardo & 3 & University of São Paulo & $\mathrm{L}$ & Callegati Franco & 3 & University of Bologna \\
\hline $\mathrm{C}$ & Kon Fabio & 3 & University of São Paulo & $\mathrm{L}$ & Giallorenzo Saverio & 2 & University of Bologna \\
\hline $\mathrm{C}$ & Milojicic Dejan & 1 & Hewlett Packard Labs & $\mathrm{L}$ & Delnevo Giovanni & 1 & University of Bologna \\
\hline $\mathrm{C}$ & Meirelles Paulo & 3 & University of São Paulo & $\mathrm{L}$ & Monti Lorenzo & 1 & University of Bologna \\
\hline $\mathrm{C}$ & Pinto Gustavo & 2 & University of São Paulo & $\mathrm{M}$ & Panichella Annibale & 4 & Delft University of Technology \\
\hline $\mathrm{D}$ & Hou Kaiyu & 3 & Northwestern University & $\mathrm{M}$ & Jan Sadeeq & 1 & Technology Peshawar Pakistan \\
\hline $\mathrm{D}$ & Wu Xiaochun & 3 & Zhejiang University & M & Arcuri Andrea & 1 & Kristiania University College \\
\hline $\mathrm{D}$ & Leng Xue & 3 & Zhejiang University & $\mathrm{M}$ & Briand Lionel & 1 & University of Ottawa \\
\hline $\mathrm{D}$ & Li Xing & 3 & University of Chicago & $\mathrm{M}$ & Olsthoorn Mitchell & 2 & Delft University of Technology \\
\hline $\mathrm{D}$ & Yu YinBo & 1 & Wuhan University & $\mathrm{M}$ & van Deursen Arie & 2 & Delft University of Technology \\
\hline $\mathrm{D}$ & Wu Bo & 3 & Google Inc. & $\mathrm{N}$ & Zimmermann Olaf & 5 & HSR University of Rapperswil \\
\hline $\mathrm{D}$ & Chen Yan & 3 & Lunghwa University & $\mathrm{N}$ & Stocker Mirko & 1 & HSR University of Rapperswil \\
\hline $\mathrm{D}$ & Yu Yinbo & 2 & Wuhan University & $\mathrm{N}$ & Zdun Uwe & 3 & University of Vienna \\
\hline $\mathrm{E}$ & Nikouei Seyed Yahya & 3 & Binghamton University & $\mathrm{N}$ & Lubke Daniel & 1 & Leibniz Universität Hannover \\
\hline $\mathrm{E}$ & Xu Ronghua & 2 & Binghamton University & $\mathrm{N}$ & Pautasso Cesare & 1 & University of Lugano \\
\hline $\mathrm{E}$ & Chen Yu & 3 & University of Singapore & $\mathrm{N}$ & Kapferer Stefan & 2 & Witten/Herdecke University \\
\hline $\mathrm{E}$ & Blasch Erik & 2 & Air Force Research Lab & $\mathrm{N}$ & Wittern Erik & 2 & Witten/Herdecke University \\
\hline $\mathrm{E}$ & Aved Alexander & 2 & US Air Force Research Lab & $\mathrm{N}$ & Leitner Philipp & 2 & University of Gothenburg \\
\hline $\mathrm{E}$ & Nagothu Deeraj & 1 & Binghamton University & $\mathrm{O}$ & Michalas Antonis & 1 & Tampere University of Technology \\
\hline $\mathrm{E}$ & Faughnan Timothy R & 1 & Binghamton University & $\mathrm{O}$ & Paladi Nicolae & 1 & Research Institutes of Sweden \\
\hline F & Sukaridhoto Sritrusta & 3 & Politeknik Surabaya & $\mathrm{O}$ & Dang Hai-Van & 3 & University of Westminster \\
\hline $\mathrm{F}$ & Panduman YY Fridelin & 1 & Politeknik Surabaya & $\mathrm{O}$ & DesLauriers James & 2 & CNRS \\
\hline $\mathrm{F}$ & Tjahjono Anang & 1 & Politeknik Surabaya & $\mathrm{O}$ & Kiss Tamas & 2 & CNRS \\
\hline $\mathrm{F}$ & Falah Muhammad Fajrul & 2 & Politeknik Surabaya & $\mathrm{O}$ & Ariyattu Resmi C & 2 & Carleton University \\
\hline $\mathrm{F}$ & Al Rasyid MU Harun & 2 & Politeknik Surabaya & $\mathrm{O}$ & Ullah Amjad & 2 & Carleton University \\
\hline $\mathrm{F}$ & Wicaksono Hendro & 2 & Politeknik Surabaya & $\mathrm{O}$ & Bowden James & 2 & Carleton University \\
\hline $\mathrm{G}$ & Kilamo Terhi & 1 & Aalto University & $\mathrm{O}$ & Krefting Dagmar & 2 & HTW Berlin \\
\hline $\mathrm{G}$ & Lwakatare Lucy Ellen & 1 & University of Helsinki & $\mathrm{O}$ & Pierantoni Gabriele & 2 & University of Westminster \\
\hline G & Karvonen Teemu & 1 & University of Helsinki & $\mathrm{O}$ & Terstyanszky Gabor & 2 & University of Westminster \\
\hline G & Heikkila & 1 & University of Oulu & $\mathrm{P}$ & Basso Tania & 1 & Universidade Estadual de Campinas \\
\hline G & Itkonen Juha & 1 & Aalto University & $\mathrm{P}$ & Antunes Nuno & 3 & University of Coimbra \\
\hline G & Kuvaja Pasi & 1 & Aalto University & $\mathrm{P}$ & Vieira Marco & 1 & University of Coimbra \\
\hline G & Mikkonen Tommi & 2 & University of Helsinki & $\mathrm{P}$ & Santos Walter & 1 & Universidade Estadual de Montes Claros \\
\hline $\mathrm{G}$ & Oivo Markku & 1 & University of Oulu & $\mathrm{P}$ & Meira Wagner & 1 & Universidade Federal de Minas Gerais \\
\hline $\mathrm{G}$ & Lassenius Casper & 1 & Aalto University & $\mathrm{P}$ & Flora Jose & 4 & University of South Carolina \\
\hline G & Kalske Miika & 1 & University of Helsinki & $\mathrm{P}$ & Goncalves Paulo & 2 & Universidade de São Paulo \\
\hline
\end{tabular}

Table 2. Cluster Authors Correspondence. 
Our analysis extracted 16 clusters from our dataset. We report in Table 2 the result of our analysis, labelling each cluster from $\mathbf{A}$ to $\mathbf{P}$. For each Cluster, we report the name of the author, the number of publications (\# pub.) in our dataset and their affiliation.

The measure gives some interesting insights. First, clusters $\mathbf{F}, \mathbf{G}, \mathbf{J}$, and $\mathbf{L}$ are totally localised in one country or the same University/Institute, they are relatively small (compared to the others in the Table), and include some of the most prolific authors ( $\mathbf{J}$ and $\mathbf{L}$ in particular). Four other clusters follow a different trend: C,H,P and $\mathbf{I}$. They are big-size clusters (respectively $6,10,8$ and 6 ), they count one core author (respectively with 3,3,4 and 3 publications) but they are rather homogeneous, the first mainly including authors from Brazil, Finland and the fourth one is from Portugal. Clusters $\mathbf{A}, \mathbf{B}, \mathbf{D}, \mathbf{K}, \mathbf{M}, \mathbf{N}$ and $\mathbf{O}$ are the most varied. Cluster $\mathbf{A}$, is the largest (22 authors) and most heterogeneous one: it includes 6 core authors from 5 different countries (Brazil, Germany, Italy, Switzerland, and the UK) and 12 co-authors from 4 countries different from those of the core authors (Australia, France, Portugal and the US). Cluster B includes 6 core authors over 24 members, distributed over just 5 countries (Brazil, Germany, Italy, Greece and Switzerland). Cluster D includes 8 authors, of which 6 are core and come both from either China or the US. Cluster $\mathbf{K}$ is another big cluster of 16 authors with include 3 core authors from the US and Germany. Clusters $\mathbf{M}, \mathbf{N}$ and $\mathbf{O}$ follow the same trend of cluster D. This means that these clusters are built around 2 core authors which represent the main affiliation provenance, respectively Holland, Germany and Switzerland, US and UK.

Overall, the communities of core authors in the dataset is distributed among three types of clusters:

- "open" clusters (A, B, D, K) of co-authors linked by a few (if not one) core authors and diverse affiliations;

- "semi-open" clusters ( $\mathbf{C}, \mathbf{G}, \mathbf{M}, \mathbf{N}$ and $\mathbf{O})$ of localised collaborators with sporadic, external collaborations;

- "closed", localised clusters $(\mathbf{F}, \mathbf{L},, \mathbf{P})$ that tend to be small but whose core authors tend to be the most prolific $(\mathbf{L})$.

Given their larger reach, semi-open and open clusters have a better chance to gather an impactful community around the topic. Our call to the authors in the field (particularly the closed clusters that tend to be prolific but rather localised) is to establish international collaborations and coordinate to foster the advancement and growth of the field.

\subsubsection{Concepts and Keywords}

We conclude our quantitative analysis by providing a graphical representation of the main keywords present in the abstract of the contributions in our dataset. To conduct our analysis, we used VOSviewer by Van Eck and Waltman (2010), a software that offers text mining functionalities for constructing and visualising co-occurrence networks of important terms extracted from a given corpus. Specifically, we ignored basic words and copyright statements and performed a full count of the words present in the text. We considered only words occurring more than fifteen times, sizing them by their relevance in terms of occurrences. The resulting graph, however, is still too large and dispersive to convey useful information: for the sake of clarity, we present here a visualisation including only the top $60 \%$ most-occurring words.

We report the visualisation of the analysis in Fig. 5.

VOSviewer automatically clustered the words in 4 areas using its modularity-based clustering algorithm, which is a variant of the cluster algorithm developed by Clauset et al. (2004) to detect communities (clusters) in a network that also considers modularity.

We can interpret the clusters as follows:

- The blue area marks the main terms of this study, grouping words like microservice and system. The result does not surprise, since those words describe the design of the systematic selection we performed.

- The green area marks technical terms as container or attack. 


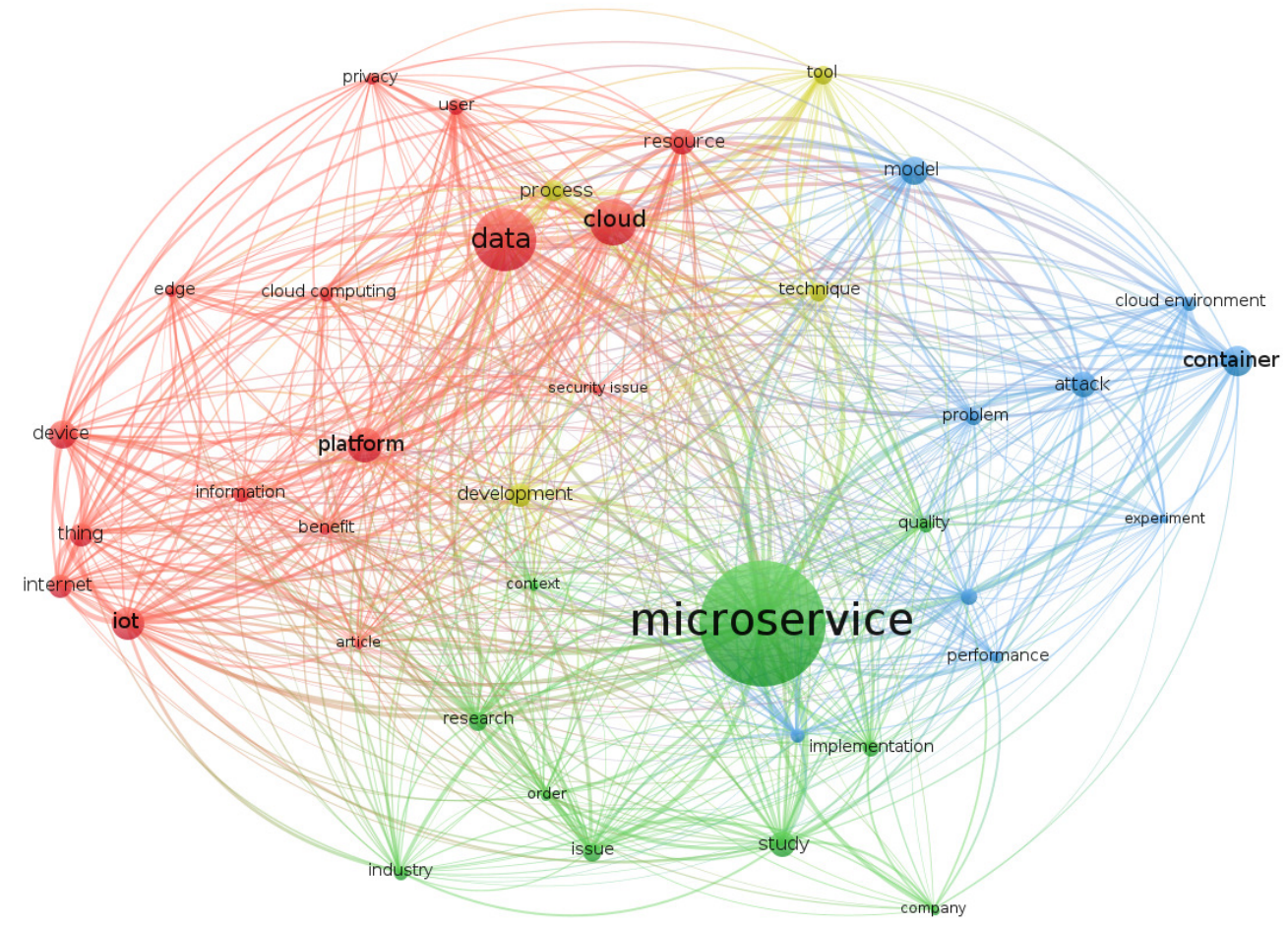

E vosviewer

Figure 5. Word-Net of the abstracts in our dataset.

- The red area identifies application terms, e.g., the targets or reasons of the research, if it is an industrial or research-focused article. We find for instance the word Internet-of-Things, as it is mainly cited with industry and research applications rather than along with terms like container and cloud.

- The yellow area includes words that identify the subject of a study, whether it be some tool, data (of the system, of the users), users, and they privacy. The word tool here is peculiar, as it acts as a bridge between the other areas. Also, this finding is somehow expected, as the field of microservice security is marked by a fairly practical orientation towards automatisation of processes and control.

\subsection{Publication Context Analysis}

In this section, we discuss trends and considerations derived from reading the selected publications and the research question detailed in Section 4.

\subsubsection{Types of Publications}

In Fig. 6 we report the distribution of the type of research contribution-whether theoretical, practical, mixed or a review.

More precisely, regarding the type of research contribution, we mapped every publication in our dataset to one of the following types:

- Theoretical for publications that present an approach for a specific problem without any implementation artefact. 


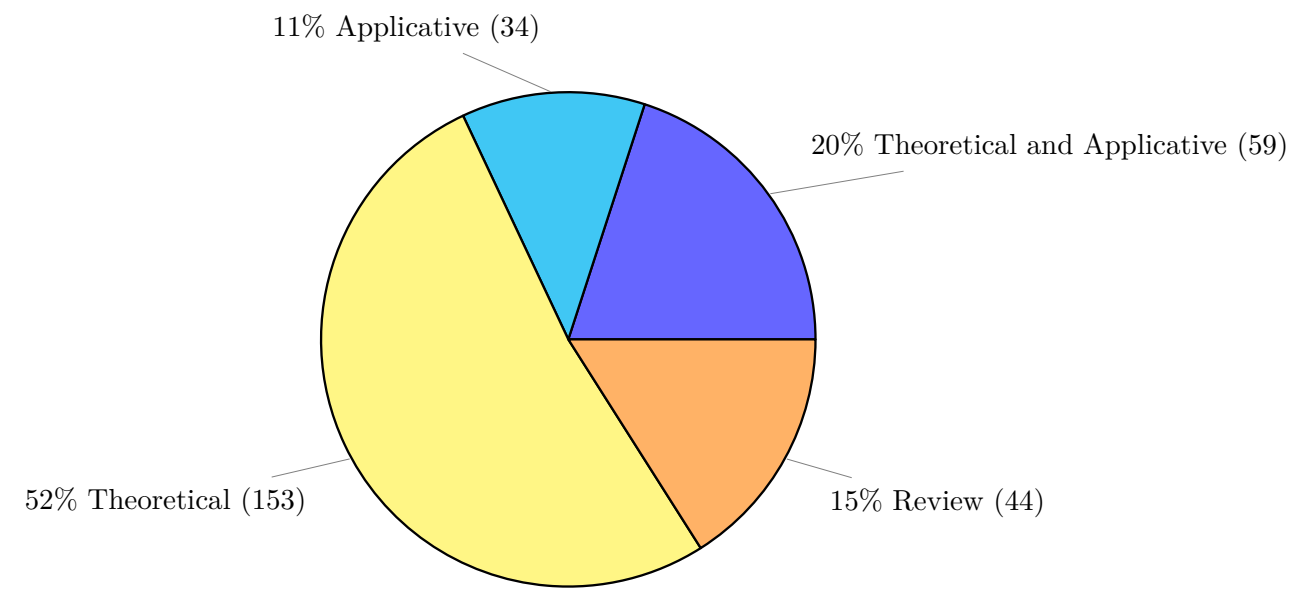

Figure 6. Type of publications.

- Applicative for publications that describe an implemented application possibly with its validation.

- Theoretical and Applicative for publications that develop a theory and provide a practical tool, framework, program, or application.

- Review for both literature reviews and social studies (e.g., on developers).

Reviews constitute $15 \%$ of the works, marking the fragmented shape of the field, which is in rapid expansion and in need of studies to map its research landscape. Besides reviews, the other contributions in the field are distributed among a 52\% share that introduces new theoretical results, a $20 \%$ share that contributes by pairing new theoretical proposals with implementations, and the remaining $11 \%$ describing pure applications. The fact that the main publications in the field are theoretical is surprising, given the prominently applied nature of microservices. Indeed, excluding reviews, we have that for every 5 publications slightly more than 3 (64\% of them) are purely theoretical. We attribute this figure to two phenomena. The first marks the current exploratory trend of the field, which is still engaged in proposing new ideas and in evaluating and maturing them into models amenable to implementation. The second phenomenon relates to the impact that microservices have at the processes/organisational level, with works that are intrinsically theoretical because their contribution can be hardly crystallised into automated implementations, e.g., for proposals of attack models or techniques for handling security within organisations and development teams. Notwithstanding the possible explanations above, it is worth noting the (quantitative) distance between contributions from academia and applications available to practitioners and the industry, which is an indicator of untapped potential for joint synergies between the two communities.

After having characterised the type of publications in the field, we proceed by exploring the results from the answer of the research questions following the 4 macro-groups presented in Section 4.

\section{Insights}

Technology transfer: the field of microservice security is still in the early phase of new idea proposals. There are just a few implementations of these ideas, which hinders industrial adoption.

\subsubsection{Threat Model}

176 publications (ca. $65 \%$ of the dataset) give a positive answer to at least one question of this category. However, only 53 publications among those 120 (ca. 30\% of the total dataset) mentioned the usage of at least one threat model to analyse or classify threats The reason for 


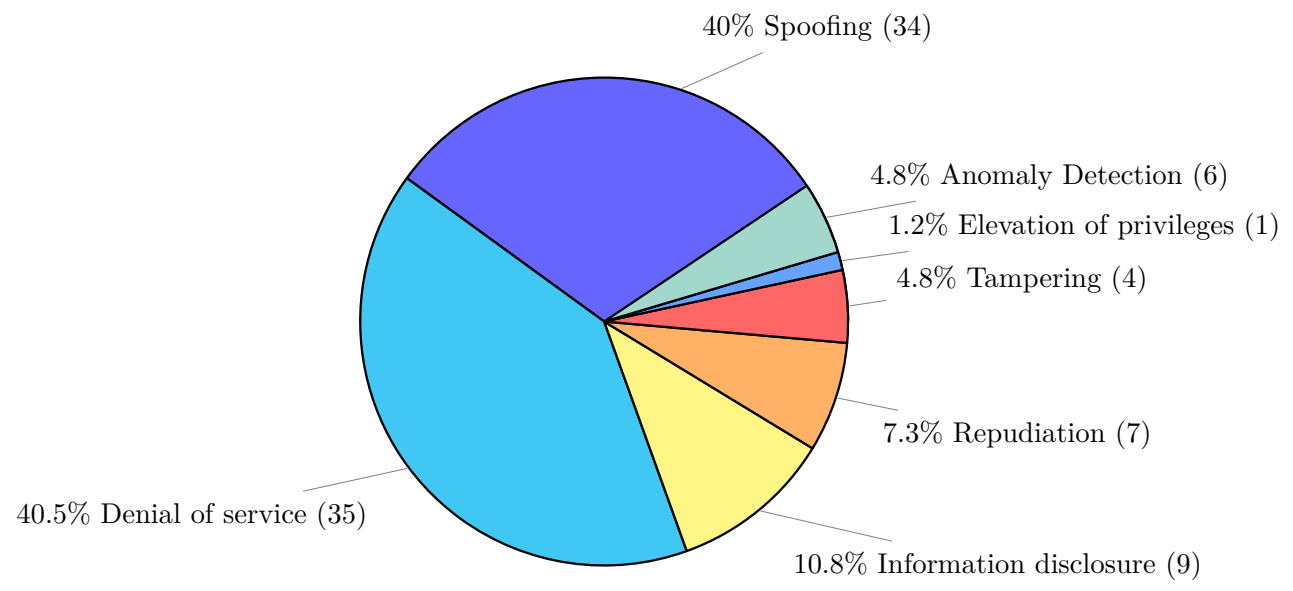

Figure 7. Attack type identified following the STRIDE classification.

those publications to adopt a threat model vary, from publications that use the model to motivate their proposed solutions to reviews that use the model to structure their overview of the state of the art. Interestingly, in ca. $80 \%$ of those publications that mention the usage of at least one known threat model, the model is tailored to work on a specific application scenario. This is an indication of the lack of usage of a generic threat model for microservice security. We conjecture that this lack of usage of generic threat models is due to the fact that the majority of research done on microservice security comes from the software (engineering, languages) side of the field, rather than from the side of security, which advocates for a security-by-design approach.

A complementary explanation of that phenomenon is that there is no affirmed threat model for microservices, e.g., due to the difficulty of making the model specific enough for microservices yet avoiding the infamous problem of threat explosion, where the effort required to prioritise and consider all threats starts exceeding the benefits of proposing methods to manage them Wuyts et al. (2018). Threat explosion is a known problem of neighbouring areas to microservices, like cloud, edge, and fog computing Di Francesco et al. (2017); Ibrahim et al. (2019); Guija and Siddiqui (2018); Lou et al. (2020); Flora (2020); Truong and Klein (2020); Russinovich et al. (2021) where the authors resorted to defining smaller, customised threat models rather than adopting standard ones, due to the problem of requiring conspicuous adaptation efforts to tailor them to such complex and multifaceted architectures.

Regarding the possible attacks addressed in the publications, Fig. 7 categorises the publications based on the STRIDE threats, following up on question Q2 asking if the publication involves at least one of threats of the STRIDE classification. The most commonly tackled attacks are of the "spoofing" and "denial of service" kinds. This is an effect of the push for fine-granularity and independence of services advocated by microservices, where applications result from several small (in size), independent software components that communicate with each other. Such decentralised communication/coordination is one of the most important attack vectors for microservice applications, in particular, the possibility to disguise a communication from an unknown source as being from a known, trusted source, which matches the spoofing attack category. Such attacks, along with tampering and repudiation ones (which together represent more then half of the attack types found in our collection), entail the need for solutions to address attacks centred around exploits of data provenance.

A similar consideration can be made for denial-of-service attacks, where the flexible scalability of microservices allows malicious intruders to, e.g., scale up peripheral microservices and hit more central and well-protected components with (distributed) overpowering attacks. 
Insights

Adoption of security-by-design: security in microservice frequently comes as an afterthought, whereas it should be one of the main concerns for their engineering. Data provenance: the quantity of spoofing, tampering, and repudiation attacks highlights the need to address the general problem of data provenance in microservices. Dedicated attack trees and threat models: while there are attacks that specifically pertain to microservices, such as those that leverage the scalability of microservice architectures to cause denial of service, there are no dedicated threat models to help developers become aware of those particular threats.

\subsubsection{Security Approach (Mitigation)}

In terms of mitigation solutions to security issues proposed by the publications (questions Q6-Q10), the most common approach (45 publications) is to address specific problems, such as authentication or exfiltration, rather than suggesting a general approach. Publications dealing with architectural aspects rarely address the overall picture (only 25, roughly $8 \%$, publications focus on IDS, IPS, Exfiltration Leaks and Threat Intelligence). Again, they focus on local threats like intra-communications or authentication (question Q11). These observations suggest that there is a lack of security approaches that address applications across the full stack.

As far as privacy and GDPR are involved (question Q12), surprisingly, only 9 publications consider privacy protection as relevant or worthy of analysis. In particular, only one publication Badii et al. (2019) considers the GDPR as a guideline to follow in order to protect the privacy of users. Examples of this kind of guideline application are shown in Voigt and Von dem Bussche (2017). Considering that many of the solutions included in the dataset are Cloud-based solutions, it is surprising to note that only one publication claims to be GDPR compliant.

Insights

Global view/control: the distributed nature of microservices introduces the need for technologies that provide global yet decentralised observability and control, i.e., tools that aid in the enforcement of security policies over a whole architecture without single points of failure.

React \& recover techniques: while we found solution to prevent and detect attacks, there are only a few proposals about how microservice systems could react to and recover from them.

Comprehensive technological references: microservices use diverse sets of technology stacks, each characterised by peculiar exploits. To secure microservice architectures effectively, implementors need dedicated technological references to avoid known threats.

\subsubsection{Infrastructure}

We start the discussion by first focusing on the type of microservice infrastructure used by the various contributions. Specifically, we have 205 publications in our dataset that answer positively to question Q13. The breakdown of the answers is:

- $39 \%$ (80) describe a centralised approach;

- $24 \%$ (49) use a decentralised approach;

- $17 \%$ (35) resort to a hybrid approach;

- $20 \%$ (41) do not specify which approach they use.

The most widely adopted turns out to be the centralised one. We conjecture two explanations behind this observation. First, the centralised approach has the merit of simplifying the definition, deployment, monitoring, and evolution of policies holding over all the components in a given architecture - traded off with scalability issues and single-point-of-failure concerns. Second, 
we note that, among the approaches that appeared early in the literature, many focused on converting monolithic applications into microservice applications. Clearly, having a centralised controller that manages the orchestration of microservices helps this process and is closer in spirit to the monolithic workflow. However, the advent of federated, multi-cloud solutions (that prevent the identification/deployment of a centralised authority over the whole peer network) as well as new distributed-consensus technologies (e.g., blockchains), has led to a decentralisation of control, making new decentralised or hybrid solutions emerge (in our dataset) starting from 2018. As an example, in 2015 and 2016, we find publications such as Callegati et al. (2016) and Lysne et al. (2016) which presented centralised approaches to enable security in microservice platforms, while starting from 2018 hybrid and decentralised solutions appear like Pahl and Donini (2018) for certificate-based authentication or Andersen et al. (2018), Andersen et al. (2017) where authors propose a decentralise high-fidelity city-scale emulation to verify the scalability of the authorisation tier.

We notice that the advent of new distributed-consensus technologies also affected the orchestration approach of microservice solutions. For example, works such as Xu et al. (2019b) propose a decentralised, blockchain-based data-access control for microservices. Recent contributions also tackled the problem of authentication and authorisation in decentralised settings, e.g., Bánáti et al. (2018) develops a workflow-oriented authorisation framework to enforce authorisation policies in a decentralised manner, Taha et al. (2019) presents a new algorithm that distribute tasks on clusters of vehicular ad-hoc networks, Zhiyi et al. (2018) proposes a secure decentralised energy management framework, and Tourani et al. (2019) describes a decentralised data-centric SECurity-as-a-Service (SECaaS) framework for elastic deployment and provisioning of security services. Another interesting work has been done in Falah et al. (2020) where authors brought the concept of a digital twin to show how a microservice infrastructure approach can speed up the process of deploy complex infrastructure components.

Infrastructure as a Service (IaaS), which is the focus of question Q14, is also a recurrent topic in our dataset, with 66 publications yielding a positive answer. IaaS include solutions that provide and manage low-level infrastructural components, like computing resources, data storage, network components, etc. We notice that IaaS is mentioned mainly as the modality used to deploy the solution but is not studied as a security subject/mechanism per se. Works such as Sultan et al. (2019) emerge as exceptions; their authors analysed the security benefits obtained using a container-based infrastructure exposed as a service.

Question Q15 investigates Service Discovery, i.e., the automatic detection of services and their functionalities available in a given architecture/network. 16 publications mention Service Discovery in the context of security. Mainly, they propose architectures that support reactive mechanisms for the detection of security issues. Of those, only 2 mention service registration procedures that include data for performing the preventive analysis of the composition, with the goal of statically finding and fixing possible vulnerabilities and misconfigurations: Callegati et al. (2018) and Kamble and Sinha (2016).

\section{Insights}

Global view/control: while there is not a definitive approach to microservice security control (whether it be centralised, decentralised, or hybrid), there is a recognised need for applying security control policies in a consistent way across all microservices belonging in the same architecture.

\subsubsection{Development}

DevOps and Agile are recurring topics in our dataset. Based on the answer to question Q16, 76 publications used the DevOps approach, while, answering to Q17, 57 used Agile methods - of those 99 publications which represent the $40 \%$ of all publications in our dataset, 10 mention both approaches. There is a common consensus in these publications that Agile/DevOps is important in security because microservices seem to be the perfect match for this type of software development model (Vehent (2018); Hsu (2018)). In particular, microservices align with the tenet of both approaches: to assign dedicated, independent teams to the development of small and 
independent components within the architecture Continuous Integration (CI) process. However, the majority of the selected publications provide no in-depth security analysis of any of the two development approaches, but rather indicate the inclusion of generic security measures in the steps of the development methods. Only three works, namely Mansfield-Devine (2018), Anisetti et al. (2019) and Kumar and Goyal (2020), propose concrete and specific variants of the DevOps approach that tackle security issues - in particular Mansfield-Devine (2018) explicitly cites the guidelines of DevSecOps Hsu (2018).

Migration is one of the main challenges faced in this context; migrating applications introduces important security concerns Lwakatare et al. (2019) that are difficult to track, due to the lack of appropriate devices (both organisational and linguistic) to elicit them from the source codebase and make sure they hold in the migrated one. Another major challenge is the coordination between development teams in the context of privacy-handling issues Gupta et al. (2019). Also, security becomes a challenging aspect since the (small, independent) teams need to know many aspects of security Leite et al. (2019) and those DevOps criteria for testing, building, and deployment automation are often neither properly followed in industrial environments Bogner et al. (2019), nor for automated scans Chondamrongkul et al. (2020).

When considering domain- and model-driven approaches (questions Q18 and Q19), 16 publications consider domain-driven approaches and 26 consider model-driven ones, such as Kapferer and Zimmermann (2020); Avritzer et al. (2020). These topics are therefore not as widespread as DevOps. Moreover, all citations in these cases are just brief references of the development approach, and lack a discussion on how one of the two approaches can be used in a security context on microservices.

The last question in this category, Q20, concerns security standards, i.e., curated sets of technologies, policies, concepts, safeguards, guidelines, assessments, procedures, training programmes that should be adopted to reduce security risks and mitigate attacks. The answers we gathered for this question surprised us. Indeed, security standards are a staple element of industries and organisations that want to impose and guarantee a certain level of security on their members and collaborators (often also for certification purposes - Stewart et al. (2012), Lie et al. (2020)). Despite their widespread use in practice, only 7 publications mention security standards. In particular, Souppaya et al. (2017) mentions the usage of X.509 to verify a secure method for key exchange between microservice. In Brenner et al. (2017) the authors show a solution for securing microservices through the SGX Intel Standard. The authors of Vassilakis et al. (2016) analyse the concept of Small-Cell-as-a-Service, i.e., a technological paradigm for the development of Virtualised Mobile Edge Computing Environments, using several mobile standards for 5G and SDN networks (e.g., MobileFlow Pentikousis et al. (2013) and VNFs Agarwal et al. (2019)). Finally, Yarygina (2018) performs a deep analysis on securing microservices, citing and analysing several know standards for both microservice management and security purposes.

\section{Insights}

Migration to microservices: there are no established techniques to help developers migrate legacy systems to microservice architectures, and in particular to identify the possible security threats that come from such a migration.

DevSecOps: agile and DevOps practices are widely used when developing microservices, yet only a few publications address how security is addressed and combined in these practices.

\subsubsection{Additional considerations}

By analysing our dataset, we were surprised to find many citations to blockchain technologies (as reported above) as well as the lack of mainstream technologies like service mesh and serverless.

Regarding blockchain technologies, we found 31 publications mentioning or explicitly using blockchains. The decentralisation and independence of microservices constitute a good pairing for the usage of blockchain technologies. Fig. 8 presents also the trend of publications using blockchain in the dataset. There is an increasing interest in blockchain applications for microservice architecture. Examples of that pairing include works such as Nagothu et al. (2018); Xu et al. 


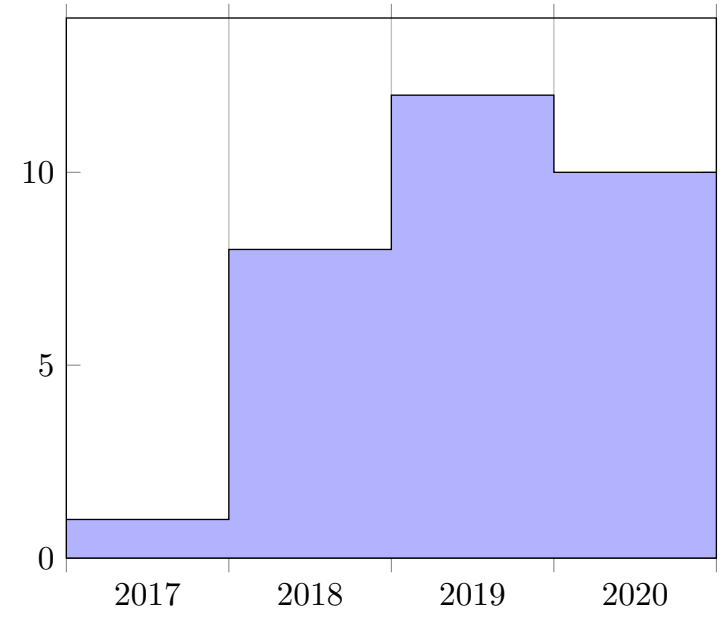

Figure 8. Blockchain trend.

(2019b), where the trust-chain of the blockchain is combined with a decentralised microservice architecture to create strong smart contract systems or Lu et al. (2021) where authors proposed a model-driven engineering approach for blockchain applications with microservice.

New approaches for microservices design and usage such as service mesh Li et al. (2019), i.e., a dedicated infrastructure layer for facilitating service-to-service communications between microservices is just mentioned by 3 works: Pahl and Donini (2018), where the authors indicate a service mesh architecture for authenticating services - securely adding information to their executables and validating the correct execution of distributed entities with such certificate-based approach - and Suneja et al. (2019), which mentions the service-mesh sidecar pattern used to control security. Another interesting work regarding service mesh is Hahn et al. (2020) where authors analysed under several scenarios issues and challenges in Service Meshes

Similarly, serverless Hendrickson et al. (2016) is mentioned only in 4 publications. We did not expect to find $(50 \%)$ more citations of serverless than those regarding service mesh. Serverless is a cloud computing execution model in which the cloud provider dynamically manages the allocation/scaling of machine resources depending on inbound requests. Indeed, while the service mesh is a technology born within the (micro)service-oriented context, serverless is a more neighbouring concept to that of stateless microservice deployment.

In this context, the most relevant publication is Casale et al. (2019), which presents the results of a European research project to develop a model-driven DevOps framework for creating and managing applications based on serverless computing. Its main result consists in designing applications as fine-grained and independent microservices that can efficiently and optimally exploit the serverless paradigm. The serverless term, despite starting to get momentum, is still loosely related to microservices.

Given their increasing importance and impact in the industry and their close relation with microservices, we argue that both service mesh and serverless will attract the general attention of the research community in the near future, as well as that of security research.

\section{Insights}

Comprehensive technological references: the progressive adoption of new technologies in the world of microservices (such as blockchains, service meshes, and serverless) calls for dedicated investigations and reports on their impact on the security of these systems.

\subsubsection{Correlation between Research Questions}

The amount of data collected in our dataset is large enough to represent a statistically-relevant sample. In this section, we leverage this to study correlations between our research questions, by 


\begin{tabular}{|c|c|c|c|c|c|c|c|c|c|c|c|c|c|c|c|c|c|c|c|}
\hline & Q2 & Q3 & Q4 & Q5 & Q6 & Q7 & Q8 & Q9 & Q10 & Q11 & 212 & Q13 & 14 & 15 & 16 & 17 & Q18 & 19 & \\
\hline Q2 & & $27.11 \%$ & $32.80 \%$ & $8.10 \%$ & $13.75 \%$ & $3.19 \%$ & $.74 \%$ & $12.36 \%$ & $0.41 \%$ & $24.68 \%$ & $-4.12 \%$ & $-22.74 \%$ & $-8.06 \%$ & $6.27 \%$ & $-3.88 \%$ & $-6.71 \%$ & $8.45 \%$ & $0.19 \%$ & $0.41 \%$ \\
\hline Q4 & $32.80 \%$ & $28.59 \%$ & & $12.18 \%$ & $6.30 \%$ & $5.05 \%$ & $10.50 \%$ & $6.05 \%$ & $8.45 \%$ & $17.28 \%$ & $9.01 \%$ & $-10.39 \%$ & $-7.34 \%$ & $-0.42 \%$ & $1.05 \%$ & $1.34 \%$ & $6.88 \%$ & $-6.90 \%$ & $.45 \%$ \\
\hline Q6 & $13.75 \%$ & $18.93 \%$ & $6.30 \%$ & $6.15 \%$ & & $77.49 \%$ & $22.89 \%$ & $14.23 \%$ & $14.86 \%$ & $3.83 \%$ & $5.58 \%$ & $0.88 \%$ & $17.76 \%$ & $14.23 \%$ & $-0.42 \%$ & $2.99 \%$ & $6.90 \%$ & $4.96 \%$ & $-5.83 \%$ \\
\hline Q7 & $3.19 \%$ & $29.11 \%$ & $5.05 \%$ & $8.26 \%$ & $77.49 \%$ & & $20.77 \%$ & $12.55 \%$ & $17.68 \%$ & $1.97 \%$ & $0.88 \%$ & $0.78 \%$ & $15.67 \%$ & $12.55 \%$ & $4.41 \%$ & $-1.48 \%$ & $10.17 \%$ & $7.44 \%$ & $-5.14 \%$ \\
\hline Q8 & $-7.74 \%$ & $7.49 \%$ & $10.50 \%$ & $12.31 \%$ & $22.89 \%$ & $20.77 \%$ & & $10.03 \%$ & $14.15 \%$ & $-5.27 \%$ & $20.31 \%$ & $20.12 \%$ & $31.15 \%$ & $13.76 \%$ & $8.28 \%$ & $9.01 \%$ & $4.83 \%$ & $-4.89 \%$ & $-8.03 \%$ \\
\hline Q11 & $24.68 \%$ & $16.69 \%$ & $17.28 \%$ & $-12.44 \%$ & $3.83 \%$ & $1.97 \%$ & $-5.27 \%$ & $3.19 \%$ & $8.88 \%$ & & $0.36 \%$ & $5.13 \%$ & $-17.71 \%$ & $0.15 \%$ & $12.62 \%$ & $9.16 \%$ & $9.02 \%$ & $6.24 \%$ & $8.88 \%$ \\
\hline Q12 & $-4.12 \%$ & $8.54 \%$ & $9.01 \%$ & $5.04 \%$ & $5.58 \%$ & $0.88 \%$ & $20.31 \%$ & $13.09 \%$ & $10.14 \%$ & $0.36 \%$ & & $-1.44 \%$ & $9.26 \%$ & $4.38 \%$ & $-1.62 \%$ & $6.16 \%$ & $1.34 \%$ & $-4.32 \%$ & $-2.81 \%$ \\
\hline Q13 & $-22.74 \%$ & $0.75 \%$ & $-10.39 \%$ & $-2.41 \%$ & $0.88 \%$ & $0.78 \%$ & $20.12 \%$ & $-0.84 \%$ & $0.37 \%$ & $5.13 \%$ & $-1.44 \%$ & & $26.24 \%$ & $9.08 \%$ & $11.22 \%$ & $13.12 \%$ & $7.16 \%$ & $5.77 \%$ & $5.29 \%$ \\
\hline Q14 & $-8.06 \%$ & $5.51 \%$ & $-7.34 \%$ & $5.31 \%$ & $17.76 \%$ & $15.67 \%$ & $31.15 \%$ & $15.70 \%$ & $7.54 \%$ & $-17.71 \%$ & $9.26 \%$ & $26.24 \%$ & & $22.90 \%$ & $10.67 \%$ & $12.47 \%$ & $11.75 \%$ & $8.50 \%$ & $-3.18 \%$ \\
\hline Q15 & $6.27 \%$ & $15.10 \%$ & $-0.42 \%$ & $9.24 \%$ & $14.23 \%$ & $12.55 \%$ & $13.76 \%$ & $14.01 \%$ & $6.04 \%$ & $0.15 \%$ & $4.38 \%$ & $9.08 \%$ & $22.90 \%$ & & $13.07 \%$ & $10.85 \%$ & $18.85 \%$ & $14.01 \%$ & $-3.80 \%$ \\
\hline Q16 & $-3.88 \%$ & $-6.93 \%$ & $1.05 \%$ & $-10.48 \%$ & $-0.42 \%$ & $4.41 \%$ & $8.28 \%$ & $-0.66 \%$ & $5.95 \%$ & $12.62 \%$ & $-1.62 \%$ & $11.22 \%$ & $10.67 \%$ & $13.07 \%$ & & $57.34 \%$ & $25.21 \%$ & $19.94 \%$ & $0.85 \%$ \\
\hline
\end{tabular}

Table 3. Correlation matrix among research questions.

way of the answers that the publications in our dataset give to each of them. Correlations can be used to understand which of the different aspects of microservice security are most commonly in a positive correlation (paired) in the dataset, and which ones are negatively correlated (mutually exclusive).

We report in Table 3 the correlation matrix - excluding research question Q1, since no publication answered it. While the obtained matrix is symmetric and we could report just one half, in Table 3 we report the full matrix for convenience, to provide a more immediate view of how each question correlates with all of the other ones.

We conditionally colour the cells of the Table, first, attributing colour intensity according to correlation absolute value - maximal intensity for $100 \%$ and degrading towards $0 \%$-, second, setting a transition threshold above $30 \%$ (absolute value) from green to orange, to help to spot relevant correlations. Looking at the Table, we notice the predominance of light-coloured cells. This result can be interpreted as an indication that the research questions used in this work are mostly orthogonal, and thus suited to cover the reviewed subject with almost no wasteful overlap.

No anti-correlation was found, i.e., negative correlations over the $30 \%$ threshold in absolute value. In the following, we comment on all positive correlations above $30 \%$.

Q2-Q4 (32,80\%) The questions relate the use of STRIDE threat model with one of is identified specific threats. This seems to be an obvious correlation since we are looking for a specific STRIDE path or at least one of his threats.

Q7-Q6 (77,49\%). The questions ask if the publication mentions IPS or IDS functionalities respectively. The strong correlation indicates how IPS and IDS are strictly related. Indeed, in practice, IDS may exist without IPS, but not the opposite, because prevention mechanisms are typically built as a reaction to a detected attack;

Q8-Q14 (31,15\%) The questions relate Threat Intelligence functionalities with Infrastructure as a Service deployment, which can define a campaign strategy for a Threat Intelligence analysis.

Q17-Q16 (57,34\%) The questions relate the Agile development practice with DevOps and Continuous Integration. As also emphasised in other studies like Lwakatare et al. (2019), this correlation can be easily explained by the fact that DevOps is sometimes considered an Agile method or its evolution. Processes adopting DevOps, therefore, adopt also Agile;

Q19-Q18 (40,00\%) The questions relate Domain-Driven Development and Model-Driven Development. We conjecture that this correlation is present because mentions of Domain- 
Driven Development often mentions Model-Driven Development as an alternative approach and vice versa;

Q18-Q17 (33,08\%) The questions relate Domain-Driven Development and Agile methods, indicating a correlation, mainly because often Agile methods employ Domain-Driven Development.

\subsection{Threats to validity}

Our study is subject to limitations that can be categorised into construct validity, external validity, internal validity, and reliability following the guidelines of Runeson et al. (2012).

Construct validity "reflects to what extent the operational measures that are studied really represent what the researcher has in mind and what is investigated according to the research questions.". To mitigate a potential misinterpretation and making sure that the constructs discussed in the interview questions are not interpreted differently by the researchers, we adopted various triangulation rounds using online meetings and we designed a set of binary research questions to foster objectivity in answering them.

Another potential risk regards whether we were exhaustive during data collection, i.e., whether we may have missed any significant publication in our review. This risk cannot be completely mitigated but to minimise this risk we deliberately chose to have simple and broad keywords giving more initial hits that later were further filtered out. Moreover, we conducted a snowballing process to extend our initial dataset looking for potentially relevant publications that our query did not select.

External validity regards the applicability of a set of results in a more general context and is not a concern for this study since we focus on the intersection of the fields of microservices and security without any attempt of generalising the findings to a broader context. We do not claim that either our qualitative or our quantitative findings should also hold for other large fields.

Internal validity is of concern when causal relations are examined when there is a risk that the investigated factor is also affected by a third factor. This thread is not a concern for this study because we presented only correlations between different factors but did not examine causal relations.

Reliability concerns to what extent the data collection and analysis depend on the actual researchers. This risk has been partially mitigated by selecting as many objective criteria as possible for the filtering and by requiring at least a two-people consensus in case of more subjective decisions. In particular, the retrieval of the publications was performed by using search engines. The first filtering of the results (Step 2, cf. Section 3) was conducted by running a script that uses objective criteria such as counting the number of keywords present and the length of the publication. These automatically computed results were double-checked by at least one author to prevent problems due to the parsing of PDFs and to make sure that the language of the publication was English. The second filtering (Step 3, cf. Section 3) performed by reading the title, abstract, and (if needed) the body of the publication, was performed in parallel by two authors. Decision conflicts were solved by discussion involving at least two authors until a consensus was reached. For the publication analysis (Step 4, cf. Section 3), due to the binary nature and formulation of the questions, the 20 research questions were answered by the author assigned to the publication. To detect possible observer bias and errors, we selected a random subset of 15 papers and had a different author answer to the research questions. The calculation of the kappa index of agreement as proposed in Cohen (1960) over the two result sets yielded a value of $\kappa=0.99998$, giving us statistical confidence over the perceived precision of questions and objectiveness of answers.

The reliability of the study is strengthened by being open and explicit about the process of data collection and analysis. For transparency, reproducibility, and reuse, we report the data used in this study at https://doi.org/10.5281/zenodo.4774894, which includes both the final dataset with the answers to all the research questions and also the set of rejected publications along with the reason for exclusion.

We also report in the Appendix each entry of our dataset and its answers to our research questions. 


\section{DISCUSSION AND FUTURE DIRECTIONS}

In this article, we presented a systematic review of the literature regarding microservice security. To conduct our research, we followed a structured approach that allowed us to gather 290 peer-reviewed publications, which, at the time of writing, constitutes the largest curated dataset on the topic.

To study our dataset, we conducted first an investigation on the metadata of the publications, which gave us some insight to map what are the publication outlets, the communities, and the key research concepts that characterise the field. Then, we performed an analysis, associating each element in our dataset to a vector of 20 different markers - presented in the form of 20 research questions.

Since our markers belong in four micro-groups (of threat-model, security, infrastructure, and development approaches), we used that partition to provide an overview of the literature through the lenses of each cluster. As a byproduct of our analysis on the content of each publication, we found concepts and topics that we did not include in our questions but that recur in multiple publications, e.g., the usage of blockchain or service-mesh technologies. To provide a more comprehensive picture of the field, we described and contextualised also these additional elements. Since our dataset forms a statistically relevant vector field, we also performed a correlation study over the components of the vectors and reported the strongest correlations (e.g., between intrusion-detection (IDS) and intrusion-prevention (IPS) systems in microservice deployments) along with possible explanations of the identified phenomena.

In the following, we draw a summary of the main open challenges that emerged from our study, which forms a call for action for the community of researchers and practitioners working in the field of microservice security and its neighbouring areas.

Data provenance: the distributed nature of microservices calls for the certification of their outputs, which other federated services receive as input and need to trust. However, there is a lack of best practices and/or standards for such a task.

Technology transfer: there exists a sensible amount of research on microservices security, but transferring those results - e.g., viable methods and tools for validation and verification - to the industry is difficult and applications are almost non-existent.

Security-by-design adoption: while many advocate for adopting security-by-design at all stages of a microservice lifecycle (from design to monitoring), there are no established references nor guidelines on how these principles can be reliably adopted in practice.

Dedicated attack trees and threat models: threats in microservice systems can come from multiple sources, from the interaction of the layers of a chosen technology stack to how microservices interact with each other - e.g., in an exclusive network, on a federated basis, on the Web, etc. Practitioners lack dedicated attack trees and threat models to help them consider and tackle the multifaceted attack surface of microservice architectures.

Comprehensive technological references: microservice development entails the use of (heterogeneous) technology stacks, whose combinations and interactions give way to exploits at different levels. These include data leakage due to host-container interactions, threats to encryption reliability due to interacting heterogeneous standards and data-format conversions, as well as surreptitious attacks through software libraries hijacking. Besides the lack of dedicated threat models, there is also a need for concrete references to secure specific technology stacks.

Migration to microservices: several works provide structures and methods to migrate legacy systems to microservices architectures. However, there are no established techniques to elicit the assumptions and invariants (e.g., on shared-memory communication, runtime environment, concurrent/interleaved database accesses, etc.) of the legacy system that the developers of the microservices must deal with-least of all considering how those factors impact the security aspects of the migrated architecture. An additional step in this direction would benefit from following principled security-by-design disciplines. 
Global view/control: the distributed nature of microservices makes it difficult to check the correct implementation of architecture-wide security policies, especially when each microservice has a dedicated security configuration. The issue is further exacerbated by the DevOps practice of having different teams deal separately with all aspects of the microservices they develop, including the implementations of their security policies. This fact highlights the need for tools that provide global overviews and guarantees on the security policies, protocols, and invariants of microservice systems.

React \& recover techniques: while the literature on preventive and detective measures against attacks abound, little has been done on how microservices should react to attacks and, as a consequence, recover their normal behaviour.

DevSecOps: Agile and DevOps practices are widely used when developing microservices, yet there is no established reference on how these approaches should integrate security in all their aspects (from team culture, management and communication to develop technologies and techniques) and into the lifecycle of microservices.

Fragmentation of outlets: researchers (and practitioners) working on microservices security do not have reference venues (neither journals nor conferences). This has at least two negative consequences. First, it makes it more difficult to gather the relevant work that constitutes the current state-of-the-art of their field - a need to which this study provides a partial solution, in the form of a snapshot of the current field landscape. Second, reference venues work also as gathering and exchange points for researchers to discuss current problems and new ideas, form interest groups, and concretise new contributions and projects to advance the knowledge in the field. Here, our call for action is at the community level, advocating for the establishment of a few reference, high-quality venues able to focus, inform, and orient the agenda of the field.

Regarding the future steps of the line of work of this contribution, we notice that here we focused our investigation on peer-reviewed publications. However, in the general field of microservices (and their security, by extension) the grey literature - which includes nonpeer-reviewed reports, working papers, government documents (e.g., those by NIST), white papers - constitutes a relevant body of knowledge that deserves separate studies. As future work, we intend to pursue an activity similar to what we presented in this work, but purposed to investigate the grey literature

\section{ACKNOWLEDGEMENTS}

Montesi was partially supported by Villum Fonden, grant no. 29518, and by Independent Research Fund Denmark, grant no. 0135-00219.

\section{REFERENCES}

Agarwal, S., Malandrino, F., Chiasserini, C. F., and De, S. (2019). Vnf placement and resource allocation for the support of vertical services in $5 \mathrm{~g}$ networks. IEEE/ACM Transactions on Networking, 27(1):433-446.

Ahmed, A. I. A., Gani, A., Ab Hamid, S. H., Abdelmaboud, A., Syed, H. J., Mohamed, R. A. A. H., and Ali, I. (2019). Service management for iot: Requirements, taxonomy, recent advances and open research challenges. IEEE Access, 7:155472-155488.

Almeida, W. H. C., de Aguiar Monteiro, L., Hazin, R. R., de Lima, A. C., and Ferraz, F. S. (2017). Survey on microservice architecture-security, privacy and standardization on cloud computing environment. ICSEA 2017, pages 199-205.

Alshuqayran, N., Ali, N., and Evans, R. (2016). A systematic mapping study in microservice architecture. In 2016 IEEE 9th International Conference on Service-Oriented Computing and Applications (SOCA), pages 44-51. 
Balalaie, A., Heydarnoori, A., and Jamshidi, P. (2016). Microservices architecture enables devops: Migration to a cloud-native architecture. Ieee Software, 33(3):42-52.

Bélair, M., Laniepce, S., and Menaud, J.-M. (2019). Leveraging kernel security mechanisms to improve container security: a survey. In Proceedings of the 14th International Conference on Availability, Reliability and Security, pages 1-6.

Casale, G., Chesta, C., Deussen, P., Di Nitto, E., Gouvas, P., Koussouris, S., Stankovski, V., Symeonidis, A., Vlassiou, V., Zafeiropoulos, A., et al. (2016). Current and future challenges of software engineering for services and applications. In Cloud Forward, pages 34-42.

Cerny, T. and Donahoo, M. J. (2016). Survey on concern separation in service integration. In International Conference on Current Trends in Theory and Practice of Informatics, pages 518-531. Springer.

Chandramouli, R. (2019). Microservices-based application systems. NIST Special Publication, 800:204.

Clauset, A., Newman, M. E., and Moore, C. (2004). Finding community structure in very large networks. Physical review E, 70(6):066111.

Cohen, J. (1960). A coefficient of agreement for nominal scales. Educational and Psychological Measurement, 20(1):37-46.

Death, D. (2017). Information security handbook: develop a threat model and incident response strategy to build a strong information security framework. Packt Publishing Ltd.

Di Francesco, P., Lago, P., and Malavolta, I. (2019). Architecting with microservices: A systematic mapping study. Journal of Systems and Software, 150:77 - 97.

Di Francesco, P., Malavolta, I., and Lago, P. (2017). Research on architecting microservices: Trends, focus, and potential for industrial adoption. In 2017 IEEE International Conference on Software Architecture (ICSA), pages 21-30. IEEE.

Dragoni, N., Giallorenzo, S., Lafuente, A. L., Mazzara, M., Montesi, F., Mustafin, R., and Safina, L. (2017). Microservices: Yesterday, Today, and Tomorrow, pages 195-216. Springer International Publishing, Cham.

Garriga, M. (2017). Towards a taxonomy of microservices architectures. In International Conference on Software Engineering and Formal Methods, pages 203-218. Springer.

Hannousse, A. and Yahiouche, S. (2020). Securing microservices and microservice architectures: A systematic mapping study.

Hendrickson, S., Sturdevant, S., Harter, T., Venkataramani, V., Arpaci-Dusseau, A. C., and Arpaci-Dusseau, R. H. (2016). Serverless computation with openlambda. In 8th $\{$ USENIX\} Workshop on Hot Topics in Cloud Computing (HotCloud 16).

Hsu, T. H.-C. (2018). Hands-On Security in DevOps: Ensure continuous security, deployment, and delivery with DevSecOps. Packt Publishing Ltd.

Joseph, C. T. and Chandrasekaran, K. (2019). Straddling the crevasse: A review of microservice software architecture foundations and recent advancements. Software: Practice and Experience, 49(10):1448-1484.

Kamble, K. G. and Sinha, A. (2016). Service level agreements and application defined security policies for application and data security registration. US Patent App. 15/191,420.

Kohnfelder, L. and Garg, P. (1999). The threats to our products. Microsoft Interface, Microsoft Corporation, 33. 
Li, W., Lemieux, Y., Gao, J., Zhao, Z., and Han, Y. (2019). Service mesh: Challenges, state of the art, and future research opportunities. In 2019 IEEE International Conference on Service-Oriented System Engineering (SOSE), pages 122-1225. IEEE.

Lichtenthäler, R., Prechtl, M., Schwille, C., Schwartz, T., Cezanne, P., and Wirtz, G. (2019). Requirements for a model-driven cloud-native migration of monolithic web-based applications. SICS Software-Intensive Cyber-Physical Systems, pages 1-12.

Lwakatare, L. E., Kilamo, T., Karvonen, T., Sauvola, T., Heikkilä, V., Itkonen, J., Kuvaja, P., Mikkonen, T., Oivo, M., and Lassenius, C. (2019). Devops in practice: A multiple case study of five companies. Information and Software Technology, 114:217-230.

Márquez, G. and Astudillo, H. (2019). Identifying availability tactics to support security architectural design of microservice-based systems. In Proceedings of the 13th European Conference on Software Architecture-Volume 2, pages 123-129.

Montesi, F. and Weber, J. (2018). From the decorator pattern to circuit breakers in microservices. In Haddad, H. M., Wainwright, R. L., and Chbeir, R., editors, Proceedings of the 33rd Annual ACM Symposium on Applied Computing, SAC 2018, Pau, France, April 09-13, 2018, pages 1733-1735. ACM.

Noura, M., Atiquzzaman, M., and Gaedke, M. (2019). Interoperability in internet of things: Taxonomies and open challenges. Mobile Networks and Applications, 24(3):796-809.

OWASP Foundation (Nov. 2020). Open web application security project (OWASP) Application Threat Modeling. https://owasp.org/www-community/Application_Threat_Modeling.

Pentikousis, K., Wang, Y., and Hu, W. (2013). Mobileflow: Toward software-defined mobile networks. IEEE Communications Magazine, 51(7):44-53.

Plaza, A. M., Díaz, J., and Pérez, J. (2018). Software architectures for health care cyberphysical systems: A systematic literature review. Journal of Software: Evolution and Process, 30(7):e1930.

Ponce, F., Soldani, J., Astudillo, H., and Brogi, A. (2021). Smells and refactorings for microservices security: A multivocal literature review.

Puliafito, C., Mingozzi, E., Longo, F., Puliafito, A., and Rana, O. (2019). Fog computing for the internet of things: A survey. ACM Transactions on Internet Technology (TOIT), 19(2):1-41.

Runeson, P., Höst, M., Rainer, A., and Regnell, B. (2012). Case Study Research in Software Engineering - Guidelines and Examples. Wiley.

Snyder, H. (2019). Literature review as a research methodology: An overview and guidelines. Journal of Business Research, 104:333 - 339.

Soldani, J. (2019). Grey literature: A safe bridge between academy and industry? ACM SIGSOFT Softw. Eng. Notes, 44(3):11-12.

Soldani, J., Tamburri, D. A., and Van Den Heuvel, W.-J. (2018). The pains and gains of microservices: A systematic grey literature review. Journal of Systems and Software, 146:215232.

Stewart, J. M., Chapple, M., and Gibson, D. (2012). CISSP: Certified Information Systems Security Professional Study Guide. John Wiley \& Sons.

Sultan, S., Ahmad, I., and Dimitriou, T. (2019). Container security: Issues, challenges, and the road ahead. IEEE Access, 7:52976-52996.

Trnka, M., Černý, T., and Stickney, N. (2018). Survey of authentication and authorization for the internet of things. Security and Communication Networks, 2018:1-17. 
UcedaVelez, T. and Morana, M. M. (2015). Risk centric threat modeling. Wiley Online Library.

Vadapalli, S. (2018). DevOps: continuous delivery, integration, and deployment with DevOps: dive into the core DevOps strategies. Packt Publishing Ltd.

Vale, A. P., Márquez, G., Astudillo, H., and Fernandez, E. B. (2019). Security mechanisms used in microservices-based systems: A systematic mapping. In CLEI, pages 1-10.

Van Eck, N. and Waltman, L. (2010). Software survey: Vosviewer, a computer program for bibliometric mapping. scientometrics, 84(2):523-538.

Vehent, J. (2018). Securing DevOps: Security in the Cloud. Manning Publications Co.

Voigt, P. and Von dem Bussche, A. (2017). The eu general data protection regulation (gdpr). A Practical Guide, 1st Ed., Cham: Springer International Publishing.

Vural, H., Koyuncu, M., and Guney, S. (2017). A systematic literature review on microservices. In Gervasi, O., Murgante, B., Misra, S., Borruso, G., Torre, C. M., Rocha, A. M. A., Taniar, D., Apduhan, B. O., Stankova, E., and Cuzzocrea, A., editors, Computational Science and Its Applications - ICCSA 2017, pages 203-217, Cham. Springer International Publishing.

Wohlin, C. (2014). Guidelines for snowballing in systematic literature studies and a replication in software engineering. In Proceedings of the 18th international conference on evaluation and assessment in software engineering, pages 1-10.

Wuyts, K., Van Landuyt, D., Hovsepyan, A., and Joosen, W. (2018). Effective and efficient privacy threat modeling through domain refinements. In Proceedings of the 33rd Annual ACM Symposium on Applied Computing, pages 1175-1178.

Yang, X., Wallom, D., Waddington, S., Wang, J., Shaon, A., Matthews, B., Wilson, M., Guo, Y., Guo, L., Blower, J. D., et al. (2014). Cloud computing in e-science: research challenges and opportunities. The Journal of Supercomputing, 70(1):408-464.

Yu, D., Jin, Y., Zhang, Y., and Zheng, X. (2019). A survey on security issues in services communication of microservices-enabled fog applications. Concurrency and Computation: Practice and Experience, 31(22):e4436.

\section{PUBLICATIONS DATASET}

Abidi, S., Essafi, M., Guegan, C. G., Fakhri, M., Witti, H., and Ghezala, H. H. B. (2019). A web service security governance approach based on dedicated micro-services. Procedia Computer Science, 159:372-386.

Adam, A. et al. (2020). The fog cloud of things: a survey on concepts, architecture, standards, tools, and applications. internet things 9 (2020).

Adedugbe, O., Benkhelifa, E., Campion, R., Al-Obeidat, F., Hani, A. B., and Uchitha, J. (2019). Leveraging cloud computing for the semantic web: review and trends. Soft Computing, pages $1-16$.

Ahmadvand, M. and Ibrahim, A. (2016). Requirements reconciliation for scalable and secure microservice (de) composition. In 2016 IEEE 24th International Requirements Engineering Conference Workshops (REW), pages 68-73. IEEE.

Ahmadvand, M., Pretschner, A., Ball, K., and Eyring, D. (2018). Integrity protection against insiders in microservice-based infrastructures: From threats to a security framework. In Federation of International Conferences on Software Technologies: Applications and Foundations, pages 573-588. Springer. 
Ahmed, A. I. A., Gani, A., Ab Hamid, S. H., Abdelmaboud, A., Syed, H. J., Mohamed, R. A. A. H., and Ali, I. (2019). Service management for iot: Requirements, taxonomy, recent advances and open research challenges. IEEE Access, 7:155472-155488.

Akkermans, S., Crispo, B., Joosen, W., and Hughes, D. (2018). Polyglot cerberos: Resource security, interoperability and multi-tenancy for iot services on a multilingual platform. In Proceedings of the 15th EAI International Conference on Mobile and Ubiquitous Systems: Computing, Networking and Services, pages 59-68.

Alaluna, M., Ferrolho, L., Figueira, J. R., Neves, N., and Ramos, F. M. (2020). Secure multi-cloud virtual network embedding. Computer Communications, 155:252-265.

Ali, I. M., Caprolu, M., and Pietro, R. D. (2020). Foundations, properties, and security applications of puzzles: A survey. ACM Computing Surveys (CSUR), 53(4):1-38.

Almeida, W. H. C., de Aguiar Monteiro, L., Hazin, R. R., de Lima, A. C., and Ferraz, F. S. (2017). Survey on microservice architecture-security, privacy and standardization on cloud computing environment. ICSEA 2017, pages 199-205.

Alulema, D., Criado, J., Iribarne, L., Fernández-García, A. J., and Ayala, R. (2020). A modeldriven engineering approach for the service integration of iot systems. Cluster Computing, 23(3):1937-1954.

Amir-Mohammadian, S. and Kari, C. (2020). Correct audit logging in concurrent systems. Electronic Notes in Theoretical Computer Science, 351:115-141.

Andersen, M. P., Kolb, J., Chen, K., Culler, D. E., and Katz, R. H. (2017). Old democratizing authority in the built environment. In Whitehouse, K., Dutta, P., and Noh, H. Y., editors, Proceedings of the 4 th ACM International Conference on Systems for Energy-Efficient Built Environments, BuildSys 2017, Delft, The Netherlands, November 08-09, 2017, pages 23:1-23:10. ACM.

Andersen, M. P., Kolb, J., Chen, K., Fierro, G., Culler, D. E., and Katz, R. (2018). Democratizing authority in the built environment. ACM Transactions on Sensor Networks (TOSN), 14(34):1-26.

Anisetti, M., Ardagna, C. A., Gaudenzi, F., and Damiani, E. (2019). A continuous certification methodology for devops. In Proceedings of the 11th International Conference on Management of Digital EcoSystems, pages 205-212.

Avritzer, A., Ferme, V., Janes, A., Russo, B., van Hoorn, A., Schulz, H., Menasché, D., and Rufino, V. (2020). Scalability assessment of microservice architecture deployment configurations: A domain-based approach leveraging operational profiles and load tests. Journal of Systems and Software, page 110564 .

Baarzi, A. F., Kesidis, G., Fleck, D., and Stavrou, A. (2020). Microservices made attack-resilient using unsupervised service fissioning. In Proceedings of the 13th European workshop on Systems Security, pages 31-36.

Baboi, M., Iftene, A., and Gîfu, D. (2019). Dynamic microservices to create scalable and fault tolerance architecture. Procedia Computer Science, 159:1035-1044.

Badii, C., Bellini, P., Difino, A., Nesi, P., Pantaleo, G., and Paolucci, M. (2019). Microservices suite for smart city applications. Sensors, 19(21):4798.

Baker, O. and Nguyen, Q. (2019). A novel approach to secure microservice architecture from owasp vulnerabilities. In CITRENZ Conference (2019).

Bánáti, A., Kail, E., Karóczkai, K., and Kozlovszky, M. (2018). Authentication and authorization orchestrator for microservice-based software architectures. In 2018 41st International Convention on Information and Communication Technology, Electronics and Microelectronics (MIPRO), pages 1180-1184. IEEE. 
Bandeira, A., Medeiros, C. A., Paixao, M., and Maia, P. H. (2019). We need to talk about microservices: an analysis from the discussions on stackoverflow. In 2019 IEEE/ACM 16th International Conference on Mining Software Repositories (MSR), pages 255-259. IEEE.

Basso, T., Moraes, R., Antunes, N., Vieira, M., Santos, W., and Meira, W. (2017). Privaaas: privacy approach for a distributed cloud-based data analytics platforms. In 2017 17th IEEE/ACM International Symposium on Cluster, Cloud and Grid Computing (CCGRID), pages 1108-1116. IEEE.

Beekman, J. G. and Porter, D. E. (2017). Challenges for scaling applications across enclaves. In Proceedings of the 2nd Workshop on System Software for Trusted Execution, pages 1-2.

Beheshti, A., Benatallah, B., Tabebordbar, A., Motahari-Nezhad, H. R., Barukh, M. C., and Nouri, R. (2019). Datasynapse: a social data curation foundry. Distributed and Parallel Databases, 37(3):351-384.

Bélair, M., Laniepce, S., and Menaud, J.-M. (2019). Leveraging kernel security mechanisms to improve container security: a survey. In Proceedings of the 14 th International Conference on Availability, Reliability and Security, pages 1-6.

Bertolino, A., Angelis, G. D., Guerriero, A., Miranda, B., Pietrantuono, R., and Russo, S. (2020). Devopret: Continuous reliability testing in devops. Journal of Software: Evolution and Process, page e2298.

Bhattacharya, R. (2019). Smart proxying for microservices. In Proceedings of the 20th International Middleware Conference Doctoral Symposium, pages 31-33.

Bogatinovski, J., Nedelkoski, S., Cardoso, J., and Kao, O. (2020). Self-supervised anomaly detection from distributed traces. In 2020 IEEE/ACM 13th International Conference on Utility and Cloud Computing (UCC), pages 342-347. IEEE.

Bogner, J., Fritzsch, J., Wagner, S., and Zimmermann, A. (2019). Microservices in industry: insights into technologies, characteristics, and software quality. In 2019 IEEE International Conference on Software Architecture Companion (ICSA-C), pages 187-195. IEEE.

Bozan, K., Lyytinen, K., and Rose, G. M. (2020). How to transition incrementally to microservice architecture. Communications of the ACM, 64(1):79-85.

Brambilla, M., Umuhoza, E., and Acerbis, R. (2017). Model-driven development of user interfaces for iot systems via domain-specific components and patterns. Journal of Internet Services and Applications, 8(1):14.

Brenner, S., Hundt, T., Mazzeo, G., and Kapitza, R. (2017). Secure cloud micro services using intel sgx. In IFIP International Conference on Distributed Applications and Interoperable Systems, pages 177-191. Springer.

Brito, A., Fetzer, C., Köpsell, S., Pietzuch, P., Pasin, M., Felber, P., Fonseca, K., Rosa, M., Gomes, L., Riella, R., et al. (2019). Secure end-to-end processing of smart metering data. Journal of Cloud Computing, 8(1):1-13.

Bromberg, Y.-D. and Gitzinger, L. (2020). Droidautoml: A microservice architecture to automate the evaluation of android machine learning detection systems. In IFIP International Conference on Distributed Applications and Interoperable Systems, pages 148-165. Springer.

Brondolin, R. and Santambrogio, M. D. (2020). A black-box monitoring approach to measure microservices runtime performance. ACM Transactions on Architecture and Code Optimization (TACO), 17(4):1-26.

Brucker, A. D., Zhou, B., Malmignati, F., Shi, Q., and Merabti, M. (2017). Modelling, validating, and ranking of secure service compositions. Software: Practice and Experience, 47(12):19231943. 
Bumblauskas, D., Mann, A., Dugan, B., and Rittmer, J. (2020). A blockchain use case in food distribution: Do you know where your food has been? International Journal of Information Management, 52:102008.

Buzachis, A. and Villari, M. (2018). Basic principles of osmotic computing: Secure and dependable microelements (mels) orchestration leveraging blockchain facilities. In 2018 IEEE/ACM International Conference on Utility and Cloud Computing Companion (UCC Companion), pages $47-52$. IEEE.

Callegati, F., Giallorenzo, S., Melis, A., and Prandini, M. (2016). Data security issues in maasenabling platforms. In 2016 IEEE 2nd International Forum on Research and Technologies for Society and Industry Leveraging a better tomorrow (RTSI), pages 1-5. IEEE.

Callegati, F., Giallorenzo, S., Melis, A., and Prandini, M. (2018). Cloud-of-things meets mobility-as-a-service: An insider threat perspective. Computers $\& 5$ Security, 74:277-295.

Camilli, M., Bellettini, C., Capra, L., and Monga, M. (2017). A formal framework for specifying and verifying microservices based process flows. In International Conference on Software Engineering and Formal Methods, pages 187-202. Springer.

Casale, G., Artač, M., van den Heuvel, W.-J., Van Hoorn, A., Jakovits, P., Leymann, F., Long, M., Papanikolaou, V., Presenza, D., Russo, A., et al. (2019). Radon: rational decomposition and orchestration for serverless computing. SICS Software-Intensive Cyber-Physical Systems, pages $1-11$.

Casale, G., Chesta, C., Deussen, P., Di Nitto, E., Gouvas, P., Koussouris, S., Stankovski, V., Symeonidis, A., Vlassiou, V., Zafeiropoulos, A., et al. (2016). Current and future challenges of software engineering for services and applications. In Cloud Forward, pages 34-42.

Casalicchio, E. and Iannucci, S. (2020). The state-of-the-art in container technologies: Application, orchestration and security. Concurrency and Computation: Practice and Experience, page e5668.

Cerny, T. and Donahoo, M. J. (2016). Survey on concern separation in service integration. In International Conference on Current Trends in Theory and Practice of Informatics, pages 518-531. Springer.

Cerny, T., Sedlisky, F., and Donahoo, M. J. (2018). On isolation-driven automated module decomposition. In Proceedings of the 2018 Conference on Research in Adaptive and Convergent Systems, pages 302-307.

Cerny, T., Svacina, J., Das, D., Bushong, V., Bures, M., Tisnovsky, P., Frajtak, K., Shin, D., and Huang, J. (2020). On code analysis opportunities and challenges for enterprise systems and microservices. IEEE Access, 8:159449-159470.

Chen, C.-A. (2019). With great abstraction comes great responsibility: Sealing the microservices attack surface. In 2019 IEEE Cybersecurity Development (SecDev), pages 144-144. IEEE.

Chen, H., Chen, P., and Yu, G. (2020). A framework of virtual war room and matrix sketch-based streaming anomaly detection for microservice systems. IEEE Access, 8:43413-43426.

Chen, J., Huang, H., and Chen, H. (2019). Informer: irregular traffic detection for containerized microservices rpc in the real world. In Proceedings of the 4th ACM/IEEE Symposium on Edge Computing, pages 389-394.

Cheruvu, S., Kumar, A., Smith, N., and Wheeler, D. M. (2020). Demystifying internet of things security: successful iot device/edge and platform security deployment. Springer Nature.

Chidambaram, N., Raj, P., Thenmozhi, K., Rajagopalan, S., and Amirtharajan, R. (2019). A cloud compatible dna coded security solution for multimedia file sharing \& storage. Multimedia Tools and Applications, 78(23):33837-33863. 
Chondamrongkul, N., Sun, J., and Warren, I. (2020). Automated security analysis for microservice architecture. In 2020 IEEE International Conference on Software Architecture Companion $(I C S A-C)$, pages 79-82. IEEE.

Ciavotta, M., Alge, M., Menato, S., Rovere, D., and Pedrazzoli, P. (2017). A microservice-based middleware for the digital factory. Procedia Manufacturing, 11:931-938.

Clancy, T. C., McGwier, R. W., and Chen, L. (2019). Post-quantum cryptography and 5g security: tutorial. In Proceedings of the 12th Conference on Security and Privacy in Wireless and Mobile Networks, pages 285-285.

Cleveland, S. B., Jamthe, A., Padhy, S., Stubbs, J., Packard, M., Looney, J., Terry, S., Cardone, R., Dahan, M., and Jacobs, G. A. (2020). Tapis api development with python: best practices in scientific rest api implementation: experience implementing a distributed stream api. In Practice and Experience in Advanced Research Computing, pages 181-187.

Copei, S., Wickert, M., and Zündorf, A. (2020). Certification as a service. In Paasivaara, M. and Kruchten, P., editors, Agile Processes in Software Engineering and Extreme Programming Workshops, pages 203-210, Cham. Springer International Publishing.

Costa, B., Pires, P. F., and Delicato, F. C. (2020). Towards the adoption of omg standards in the development of soa-based iot systems. Journal of Systems and Software, 169:110720.

da Silva, M. S. L., de Oliveira Silva, F. F., and Brito, A. (2019). Squad: A secure, simple storage service for sgx-based microservices. In 2019 9th Latin-American Symposium on Dependable Computing $(L A D C)$, pages 1-9. IEEE.

Damis, H. A., Shehada, D., Fachkha, C., Gawanmeh, A., and Al-Karaki, J. N. (2020). A microservices architecture for ads-b data security using blockchain. In 2020 3rd International Conference on Signal Processing and Information Security (ICSPIS), pages 1-4. IEEE.

Dash, P. B., Nayak, J., Naik, B., Oram, E., and Islam, S. H. (2020). Model based iot security framework using multiclass adaptive boosting with smote. Security and Privacy, 3(5):e112.

de Araujo Zanella, A. R., da Silva, E., and Albini, L. C. P. (2020). Security challenges to smart agriculture: Current state, key issues, and future directions. Array, page 100048.

De Donno, M., Giaretta, A., Dragoni, N., Bucchiarone, A., and Mazzara, M. (2019). Cyber-storms come from clouds: Security of cloud computing in the iot era. Future Internet, 11(6):127.

de Oliveira Rosa, T., Daniel, J. F. L., Guerra, E. M., and Goldman, A. (2020). A method for architectural trade-off analysis based on patterns: Evaluating microservices structural attributes. In Proceedings of the European Conference on Pattern Languages of Programs 2020, pages 1-8.

de Sousa, P. S., Nogueira, N. P., dos Santos, R. C., Maia, P. H. M., and de Souza, J. T. (2020). Building a prototype based on microservices and blockchain technologies for notary's office: An academic experience report. In 2020 IEEE International Conference on Software Architecture Companion (ICSA-C), pages 122-129. IEEE.

de Toledo, S. S., Martini, A., and Sjøberg, D. I. (2020). Improving agility by managing shared libraries in microservices. In International Conference on Agile Software Development, pages 195-202. Springer.

Delicato, F. C., Al-Anbuky, A., Kevin, I., and Wang, K. (2020). Smart cyber-physical systems: Toward pervasive intelligence systems.

Demoulin, H. M., Vaidya, T., Pedisich, I., DiMaiolo, B., Qian, J., Shah, C., Zhang, Y., Chen, A., Haeberlen, A., Loo, B. T., et al. (2018). Dedos: Defusing dos with dispersion oriented software. In Proceedings of the 34th Annual Computer Security Applications Conference, pages 712-722. 
DesLauriers, J., Kiss, T., Ariyattu, R. C., Dang, H.-V., Ullah, A., Bowden, J., Krefting, D., Pierantoni, G., and Terstyanszky, G. (2020). Cloud apps to-go: Cloud portability with tosca and micado. Concurrency and Computation: Practice and Experience, page e6093.

Dewanta, F. (2020). Secure microservices deployment for fog computing services in a remote office. In 2020 3rd International Conference on Information and Communications Technology (ICOIACT), pages 425-430. IEEE.

Di Ciccio, C., Cecconi, A., Dumas, M., García-Bañuelos, L., López-Pintado, O., Lu, Q., Mendling, J., Ponomarev, A., Tran, A. B., and Weber, I. (2019). Blockchain support for collaborative business processes. Informatik Spektrum, 42(3):182-190.

Di Francesco, P., Malavolta, I., and Lago, P. (2017). Research on architecting microservices: Trends, focus, and potential for industrial adoption. In 2017 IEEE International Conference on Software Architecture (ICSA), pages 21-30. IEEE.

Di Salle, A., Gallo, F., and Pompilio, C. (2016). Composition of advanced $(\mu)$ services for the next generation of the internet of things. In Federation of International Conferences on Software Technologies: Applications and Foundations, pages 436-444. Springer.

Di Sanzo, P., Avresky, D. R., and Pellegrini, A. (2021). Autonomic rejuvenation of cloud applications as a countermeasure to software anomalies. Software: Practice and Experience, $51(1): 46-71$.

Díaz-Sánchez, D., Marín-Lopez, A., Almenárez Mendoza, F., and Arias Cabarcos, P. (2019). Dns/dane collision-based distributed and dynamic authentication for microservices in iot. Sensors, 19(15):3292.

Diekmann, C., Naab, J., Korsten, A., and Carle, G. (2018). Agile network access control in the container age. IEEE Transactions on Network and Service Management, 16(1):41-55.

Dilshan, D., Piumika, S., Rupasinghe, C., Perera, I., and Siriwardena, P. (2020). Mschain: Blockchain based decentralized certificate transparency for microservices. In 2020 Moratuwa Engineering Research Conference (MERCon), pages 1-6. IEEE.

Du, D., Yu, T., Xia, Y., Zang, B., Yan, G., Qin, C., Wu, Q., and Chen, H. (2020). Catalyzer: Sub-millisecond startup for serverless computing with initialization-less booting. In Proceedings of the Twenty-Fifth International Conference on Architectural Support for Programming Languages and Operating Systems, pages 467-481.

Du, Q., Xie, T., and He, Y. (2018). Anomaly detection and diagnosis for container-based microservices with performance monitoring. In International Conference on Algorithms and Architectures for Parallel Processing, pages 560-572. Springer.

Elsayed, M. and Zulkernine, M. (2019). Offering security diagnosis as a service for cloud saas applications. Journal of information security and applications, 44:32-48.

Esparrachiari, S., Reilly, T., and Rentz, A. (2018). Tracking and controlling microservice dependencies. Queue, 16(4):44-65.

Esposito, C., Castiglione, A., Tudorica, C.-A., and Pop, F. (2017). Security and privacy for cloud-based data management in the health network service chain: a microservice approach. IEEE Communications Magazine, 55(9):102-108.

Fahmideh, M. and Zowghi, D. (2020). An exploration of iot platform development. Information Systems, 87:101409.

Falah, M. F., Sukaridhoto, S., Al Rasyid, M. U. H., and Wicaksono, H. (2020). Design of virtual engineering and digital twin platform as implementation of cyber-physical systems. Procedia Manufacturing, 52:331-336. 
Fetzer, C., Mazzeo, G., Oliver, J., Romano, L., and Verburg, M. (2017). Integrating reactive cloud applications in sereca. In Proceedings of the 12th International Conference on Availability, Reliability and Security, pages 1-8.

Flora, J. (2020). Improving the security of microservice systems by detecting and tolerating intrusions. In 2020 IEEE International Symposium on Software Reliability Engineering Workshops (ISSREW), pages 131-134. IEEE.

Flora, J., Gonçalves, P., and Antunes, N. (2020). Using attack injection to evaluate intrusion detection effectiveness in container-based systems. In 2020 IEEE 25th Pacific Rim International Symposium on Dependable Computing (PRDC), pages 60-69. IEEE.

Forti, S., Ferrari, G.-L., and Brogi, A. (2020). Secure cloud-edge deployments, with trust. Future Generation Computer Systems, 102:775-788.

Garg, S. and Garg, S. (2019). Automated cloud infrastructure, continuous integration and continuous delivery using docker with robust container security. In 2019 IEEE Conference on Multimedia Information Processing and Retrieval (MIPR), pages 467-470. IEEE.

George, V. M. and Mahmoud, Q. H. (2017). Claimsware: A claims-based middleware for securing iot services. In 2017 IEEE 41st Annual Computer Software and Applications Conference (COMPSAC), volume 1, pages 649-654. IEEE.

Gerking, C. and Schubert, D. (2019). Component-based refinement and verification of informationflow security policies for cyber-physical microservice architectures. In 2019 IEEE International Conference on Software Architecture (ICSA), pages 61-70. IEEE.

Gerostathopoulos, I., Bures, T., et al. (2020). A toolbox for realtime timeseries anomaly detection. In 2020 IEEE International Conference on Software Architecture Companion (ICSA-C), pages 278-281. IEEE.

Ghayyur, S. A. K., Razzaq, A., Ullah, S., and Ahmed, S. (2018). Matrix clustering based migration of system application to microservices architecture. INTERNATIONAL JOURNAL OF ADVANCED COMPUTER SCIENCE AND APPLICATIONS, 9(1):284-296.

Ghuge, S. S., Kumar, N., Savitha, S., and Suraj, V. (2020). Multilayer technique to secure data transfer in private cloud for saas applications. In 2020 2nd International Conference on Innovative Mechanisms for Industry Applications (ICIMIA), pages 646-651. IEEE.

Giaimo, F., Andrade, H., and Berger, C. (2020). Continuous experimentation and the cyberphysical systems challenge: An overview of the literature and the industrial perspective. Journal of Systems and Software, 170:110781.

Gorige, D., Al-Masri, E., Kanzhelev, S., and Fattah, H. (2020). Privacy-risk detection in microservices composition using distributed tracing. In 2020 IEEE Eurasia Conference on IOT, Communication and Engineering (ECICE), pages 250-253. IEEE.

Guija, D. and Siddiqui, M. S. (2018). Identity and access control for micro-services based $5 \mathrm{~g}$ nfv platforms. In Proceedings of the 13th International Conference on Availability, Reliability and Security, pages 1-10.

Gupta, R. K., Venkatachalapathy, M., and Jeberla, F. K. (2019). Challenges in adopting continuous delivery and devops in a globally distributed product team: a case study of a healthcare organization. In 2019 ACM/IEEE 14th International Conference on Global Software Engineering (ICGSE), pages 30-34. IEEE.

Hahn, D. A., Davidson, D., and Bardas, A. G. (2020). Mismesh: Security issues and challenges in service meshes. In International Conference on Security and Privacy in Communication Systems, pages 140-151. Springer. 
Hajek, J., Rashid, M., Sevil, M., Cinar, A., Alvarez Fernandez, P. A., and Jain, D. (2020). The necessity of interdisciplinary software development for building viable research platforms: Case study in automated drug delivery in diabetes. In Proceedings of the 21st Annual Conference on Information Technology Education, pages 390-396.

Han, J., Kim, S., Kim, T., and Han, D. (2019). Toward scaling hardware security module for emerging cloud services. In Proceedings of the 4 th Workshop on System Software for Trusted Execution, pages 1-6.

Hang, L., Ullah, I., and Kim, D.-H. (2020). A secure fish farm platform based on blockchain for agriculture data integrity. Computers and Electronics in Agriculture, 170:105251.

Haque, M. U., Iwaya, L. H., and Babar, M. A. (2020). Challenges in docker development: A large-scale study using stack overflow. In Proceedings of the 14th ACM/IEEE International Symposium on Empirical Software Engineering and Measurement (ESEM), pages 1-11.

Hasan, M. and Starly, B. (2020). Decentralized cloud manufacturing-as-a-service (cmaas) platform architecture with configurable digital assets. Journal of Manufacturing Systems, 56:157-174.

He, X. and Yang, X. (2017). Authentication and authorization of end user in microservice architecture. In Journal of Physics: Conference Series, volume 910, page 012060. IOP Publishing.

Hole, J. K. (2016). Anti-fragile ICT systems. Springer-Verlag GmbH.

Ibrahim, A., Bozhinoski, S., and Pretschner, A. (2019). Attack graph generation for microservice architecture. In Proceedings of the 34th ACM/SIGAPP Symposium on Applied Computing, pages $1235-1242$.

Iraqi, O. and El Bakkali, H. (2020). Immunizer: A scalable loosely-coupled self-protecting software framework using adaptive microagents and parallelized microservices. In 2020 IEEE 29th International Conference on Enabling Technologies: Infrastructure for Collaborative Enterprises (WETICE), pages 24-27. IEEE.

Islam, T., Manivannan, D., and Zeadally, S. (2016). A classification and characterization of security threats in cloud computing. Int. J. Next-Gener. Comput, 7(1).

Jan, S., Panichella, A., Arcuri, A., and Briand, L. (2019). Search-based multi-vulnerability testing of xml injections in web applications. Empirical Software Engineering, 24(6):3696-3729.

Jander, K., Braubach, L., and Pokahr, A. (2018). Defense-in-depth and role authentication for microservice systems. Procedia computer science, 130:456-463.

Jander, K., Braubach, L., and Pokahr, A. (2019). Practical defense-in-depth solution for microservice systems. Journal of Ubiquitous Systems $\mathcal{E}$ Pervasive Networks, 11(1):17-25.

Janjua, K., Shah, M. A., Almogren, A., Khattak, H. A., Maple, C., and Din, I. U. (2020). Proactive forensics in iot: privacy-aware log-preservation architecture in fog-enabled-cloud using holochain and containerization technologies. Electronics, 9(7):1172.

Javed, A., Robert, J., Heljanko, K., and Främling, K. (2020). Iotef: A federated edge-cloud architecture for fault-tolerant iot applications. Journal of Grid Computing, pages 1-24.

Jaworski, J., Karwowski, W., and Rusek, M. (2019). Microservice-based cloud application ported to unikernels: Performance comparison of different technologies. In International Conference on Information Systems Architecture and Technology, pages 255-264. Springer.

Jin, H., Li, Z., Zou, D., and Yuan, B. (2019). Dseom: A framework for dynamic security evaluation and optimization of mtd in container-based cloud. IEEE Transactions on Dependable and Secure Computing. 
Jin, M., Lv, A., Zhu, Y., Wen, Z., Zhong, Y., Zhao, Z., Wu, J., Li, H., He, H., and Chen, F. (2020). An anomaly detection algorithm for microservice architecture based on robust principal component analysis. IEEE Access.

Jita, H. and Pieterse, V. (2018). A framework to apply the internet of things for medical care in a home environment. In Proceedings of the 2018 International Conference on Cloud Computing and Internet of Things, pages 45-54.

Kallergis, D., Garofalaki, Z., Katsikogiannis, G., and Douligeris, C. (2020). Capodaz: A containerised authorisation and policy-driven architecture using microservices. Ad Hoc Networks, 104:102153.

Kalske, M., Mäkitalo, N., and Mikkonen, T. (2017). Challenges when moving from monolith to microservice architecture. In International Conference on Web Engineering, pages 32-47. Springer.

Kang, M., Shin, J.-S., and Kim, J. (2019). Protected coordination of service mesh for containerbased 3-tier service traffic. In 2019 International Conference on Information Networking (ICOIN), pages 427-429. IEEE.

Kang, R., Zhou, Z., Liu, J., Zhou, Z., and Xu, S. (2018). Distributed monitoring system for microservices-based iot middleware system. In International Conference on Cloud Computing and Security, pages 467-477. Springer.

Kapferer, S. and Zimmermann, O. (2020). Domain-driven service design. In Symposium and Summer School on Service-Oriented Computing, pages 189-208. Springer.

Kathiravelu, P., Van Roy, P., and Veiga, L. (2019). Sd-cps: software-defined cyber-physical systems. taming the challenges of cps with workflows at the edge. Cluster Computing, 22(3):661677 .

Ke, H., Wu, H., and Yang, D. (2020). Towards evolving security requirements of industrial internet: A layered security architecture solution based on data transfer techniques. In Proceedings of the 2020 International Conference on Cyberspace Innovation of Advanced Technologies, pages 504-511.

Kelbert, F., Gregor, F., Pires, R., Köpsell, S., Pasin, M., Havet, A., Schiavoni, V., Felber, P., Fetzer, C., and Pietzuch, P. (2017). Securecloud: Secure big data processing in untrusted clouds. In Design, Automation 83 Test in Europe Conference 85 Exhibition (DATE), 2017, pages 282-285. IEEE.

Khan, A. A. and Shameem, M. (2020). Multicriteria decision-making taxonomy for devops challenging factors using analytical hierarchy process. Journal of Software: Evolution and Process, 32(10):e2263.

Kochovski, P., Gec, S., Stankovski, V., Bajec, M., and Drobintsev, P. D. (2019). Trust management in a blockchain based fog computing platform with trustless smart oracles. Future Generation Computer Systems, 101:747-759.

Krämer, M., Frese, S., and Kuijper, A. (2019). Implementing secure applications in smart city clouds using microservices. Future Generation Computer Systems, 99:308-320.

Krishnan, P., Duttagupta, S., and Achuthan, K. (2019). Sdn/nfv security framework for fog-to-things computing infrastructure. Software: Practice and Experience.

Kumar, R. and Goyal, R. (2020). Modeling continuous security: A conceptual model for automated devsecops using open-source software over cloud (adoc). Computers $\& 3$ Security, 97:101967.

Kwon, S., Son, S.-J., Choi, Y., and Lee, J.-H. (2020). Protocol fuzzing to find security vulnerabilities of rabbitmq. Concurrency and Computation: Practice and Experience, page e6012. 
Lakhan, A. and Li, X. (2020). Transient fault aware application partitioning computational offloading algorithm in microservices based mobile cloudlet networks. Computing, 102(1):105139.

Łaskawiec, S., Choraś, M., and Kozik, R. (2019). New solutions for exposing clustered applications deployed in the cloud. Cluster Computing, 22(3):829-838.

Leite, A. F., Alves, V., Rodrigues, G. N., Tadonki, C., Eisenbeis, C., and de Melo, A. C. M. A. (2017). Dohko: an autonomic system for provision, configuration, and management of inter-cloud environments based on a software product line engineering method. Cluster Computing, 20(3):1951-1976.

Leite, L., Kon, F., Pinto, G., and Meirelles, P. (2020). Platform teams: An organizational structure for continuous delivery. In Proceedings of the IEEE/ACM 42nd International Conference on Software Engineering Workshops, pages 505-511.

Leite, L., Rocha, C., Kon, F., Milojicic, D., and Meirelles, P. (2019). A survey of devops concepts and challenges. ACM Computing Surveys (CSUR), 52(6):1-35.

Lenarduzzi, V., Lomio, F., Saarimäki, N., and Taibi, D. (2020). Does migrating a monolithic system to microservices decrease the technical debt? Journal of Systems and Software, page 110710

Li, H., Hu, H., Gu, G., Ahn, G.-J., and Zhang, F. (2018). vnids: Towards elastic security with safe and efficient virtualization of network intrusion detection systems. In Proceedings of the 2018 ACM SIGSAC Conference on Computer and Communications Security, pages 17-34.

Li, S., Xu, Q., Hou, P., Chen, X., Wang, Y., Zhang, H., and Rong, G. (2020). Exploring the challenges of developing and operating consortium blockchains: A case study. In Proceedings of the Evaluation and Assessment in Software Engineering, pages 398-404.

Li, Z., Jin, H., Zou, D., and Yuan, B. (2019). Exploring new opportunities to defeat low-rate ddos attack in container-based cloud environment. IEEE Transactions on Parallel and Distributed Systems.

Liang, X. and Zhao, Q. (2020). On the design of a blockchain-based student quality assessment system. In 2020 International Conference on High Performance Big Data and Intelligent Systems (HPBDEIS), pages 1-7. IEEE.

Lichtenthäler, R., Prechtl, M., Schwille, C., Schwartz, T., Cezanne, P., and Wirtz, G. (2019). Requirements for a model-driven cloud-native migration of monolithic web-based applications. SICS Software-Intensive Cyber-Physical Systems, pages 1-12.

Lie, M. F., Sánchez-Gordón, M., and Colomo-Palacios, R. (2020). Devops in an iso 13485 regulated environment: A multivocal literature review. In Proceedings of the 14th ACM/IEEE International Symposium on Empirical Software Engineering and Measurement (ESEM), pages $1-11$.

Liu, P., Xu, H., Ouyang, Q., Jiao, R., Chen, Z., Zhang, S., Yang, J., Mo, L., Zeng, J., Xue, W., et al. (2020). Unsupervised detection of microservice trace anomalies through service-level deep bayesian networks. In 2020 IEEE 31st International Symposium on Software Reliability Engineering (ISSRE), pages 48-58. IEEE.

Lou, P., Lu, G., Jiang, X., Xiao, Z., Hu, J., and Yan, J. (2020). Cyber intrusion detection through association rule mining on multi-source logs. Applied Intelligence, pages 1-15.

Lu, D., Huang, D., Walenstein, A., and Medhi, D. (2017). A secure microservice framework for iot. In 2017 IEEE Symposium on Service-Oriented System Engineering (SOSE), pages 9-18. IEEE. 
Lu, Q., Binh Tran, A., Weber, I., O'Connor, H., Rimba, P., Xu, X., Staples, M., Zhu, L., and Jeffery, R. (2021). Integrated model-driven engineering of blockchain applications for business processes and asset management. Software: Practice and Experience, 51(5):1059-1079.

Luntovskyy, A. and Shubyn, B. (2020). Highly-distributed systems based on micro-services and their construction paradigms. In 2020 IEEE 15th International Conference on Advanced Trends in Radioelectronics, Telecommunications and Computer Engineering (TCSET), pages 7-14. IEEE.

Luo, X., Ren, F., and Zhang, T. (2018). High performance userspace networking for containerized microservices. In International Conference on Service-Oriented Computing, pages 57-72. Springer.

Lwakatare, L. E., Kilamo, T., Karvonen, T., Sauvola, T., Heikkilä, V., Itkonen, J., Kuvaja, P., Mikkonen, T., Oivo, M., and Lassenius, C. (2019). Devops in practice: A multiple case study of five companies. Information and Software Technology, 114:217-230.

Lysne, O., Hole, K. J., Otterstad, C., Ytrehus, Ø., Aarseth, R., and Tellnes, J. (2016). Vendor malware: detection limits and mitigation. Computer, 49(8):62-69.

Ma, M., Xu, J., Wang, Y., Chen, P., Zhang, Z., and Wang, P. (2020). Automap: Diagnose your microservice-based web applications automatically. In Proceedings of The Web Conference 2020, pages 246-258.

Maati, B. and Saidouni, D. E. (2020). Ciotas protocol: Cloudiot available services protocol through autonomic computing against distributed denial of services attacks. Journal of Ambient Intelligence and Humanized Computing, pages 1-30.

Mann, Z. A. (2020). Secure software placement and configuration. Future Generation Computer Systems, 110:243-253.

Mansfield-Devine, S. (2018). Devops: finding room for security. Network Security, 2018(7):15-20.

Manu, A., Patel, J. K., Akhtar, S., Agrawal, V., and Murthy, K. B. S. (2016). Docker container security via heuristics-based multilateral security-conceptual and pragmatic study. In 2016 International Conference on Circuit, Power and Computing Technologies (ICCPCT), pages 1-14. IEEE.

Marchal, X., Cholez, T., and Festor, O. (2018). $\mu$ ndn: an orchestrated microservice architecture for named data networking. In Proceedings of the 5th ACM Conference on Information-Centric Networking, pages 12-23.

Márquez, G. and Astudillo, H. (2019). Identifying availability tactics to support security architectural design of microservice-based systems. In Proceedings of the 13th European Conference on Software Architecture-Volume 2, pages 123-129.

Melis, A., Mirri, S., Prandi, C., Prandini, M., Salomoni, P., and Callegati, F. (2018). Integrating personalized and accessible itineraries in maas ecosystems through microservices. Mobile Networks and Applications, 23(1):167-176.

Mishra, A. and Otaiwi, Z. (2020). Devops and software quality: A systematic mapping. Computer Science Review, 38:100308.

Mohamed, M. A., Challenger, M., and Kardas, G. (2020). Applications of model-driven engineering in cyber-physical systems: a systematic mapping study. Journal of Computer Languages, 59:100972.

Mohammed, T. A. and Mohammed, A. B. (2020). Security architectures for sensitive data in cloud computing. In Proceedings of the 6th International Conference on Engineering 83 MIS 2020, pages $1-6$. 
Mohsin, A. and Janjua, N. K. (2018). A review and future directions of soa-based software architecture modeling approaches for system of systems. Service Oriented Computing and Applications, 12(3-4):183-200.

Moreira, J. B., Mamede, H., Pereira, V., and Sousa, B. (2020). Next generation of microservices for the 5g service-based architecture. International Journal of Network Management, 30(6):e2132.

Morris, J. B. (2017). 10 rules for an unhackable data vault. Ubiquity, 2017(May):1-10.

Moura, J. and Hutchison, D. (2020). Fog computing systems: State of the art, research issues and future trends, with a focus on resilience. Journal of Network and Computer Applications, page 102784 .

Nagendra, V., Yegneswaran, V., Porras, P., and Das, S. R. (2019). Coordinated dataflow protection for ultra-high bandwidth science networks. In Proceedings of the 35th Annual Computer Security Applications Conference, pages 568-583.

Nagothu, D., Xu, R., Nikouei, S. Y., and Chen, Y. (2018). A microservice-enabled architecture for smart surveillance using blockchain technology. In 2018 IEEE International Smart Cities Conference (ISC2), pages 1-4. IEEE.

Nehme, A., Jesus, V., Mahbub, K., and Abdallah, A. (2018). Fine-grained access control for microservices. In International Symposium on Foundations and Practice of Security, pages 285-300. Springer.

Nehme, A., Jesus, V., Mahbub, K., and Abdallah, A. (2019). Securing microservices. IT Professional, 21(1):42-49.

Nguyen, Q. and Baker, O. (2019). Applying spring security framework and oauth2 to protect microservice architecture api. Journal of Software, pages 257-264.

Niazi, M., Mishra, A., and Gill, A. Q. (2018). What do software practitioners really think about software process improvement project success? an exploratory study. Arabian Journal for Science and Engineering, 43(12):7719-7735.

Niknejad, N., Ismail, W., Ghani, I., Nazari, B., Bahari, M., et al. (2020). Understanding service-oriented architecture (soa): A systematic literature review and directions for further investigation. Information Systems, 91:101491.

Nikolakis, N., Marguglio, A., Veneziano, G., Greco, P., Panicucci, S., Cerquitelli, T., Macii, E., Andolina, S., and Alexopoulos, K. (2020). A microservice architecture for predictive analytics in manufacturing. Procedia Manufacturing, 51:1091-1097.

Nikoloudakis, Y., Pallis, E., Mastorakis, G., Mavromoustakis, C. X., Skianis, C., and Markakis, E. K. (2019). Vulnerability assessment as a service for fog-centric ict ecosystems: A healthcare use case. Peer-to-Peer Networking and Applications, 12(5):1216-1224.

Nikouei, S. Y., Chen, Y., Aved, A., Blasch, E., and Faughnan, T. R. (2019). I-safe: Instant suspicious activity identification at the edge using fuzzy decision making. In Proceedings of the 4th ACM/IEEE Symposium on Edge Computing, pages 101-112.

Nkomo, P. and Coetzee, M. (2019). Development activities, tools and techniques of secure microservices compositions. In International Conference on Information Security Practice and Experience, pages 423-433. Springer.

Noura, M., Atiquzzaman, M., and Gaedke, M. (2019). Interoperability in internet of things: Taxonomies and open challenges. Mobile Networks and Applications, 24(3):796-809.

Olsthoorn, M., van Deursen, A., and Panichella, A. (2020). Generating highly-structured input data by combining search-based testing and grammar-based fuzzing. In 2020 35th IEEE/ACM International Conference on Automated Software Engineering (ASE), pages 1224-1228. IEEE. 
Oppermann, A., Toro, F. G., Thiel, F., and Seifert, J.-P. (2018). Secure cloud computing: Reference architecture for measuring instrument under legal control. Security and Privacy, $1(3): \mathrm{e} 18$.

Osman, A., Bruckner, P., Salah, H., Fitzek, F. H., Strufe, T., and Fischer, M. (2019a). Sandnet: Towards high quality of deception in container-based microservice architectures. In ICC 2019-2019 IEEE International Conference on Communications (ICC), pages 1-7. IEEE.

Osman, A., Hanisch, S., and Strufe, T. (2019b). Seconetbench: A modular framework for secure container networking benchmarks. In 2019 IEEE European Symposium on Security and Privacy Workshops (EuroS\&3PW), pages 21-28. IEEE.

Otterstad, C. and Yarygina, T. (2017). Low-level exploitation mitigation by diverse microservices. In European Conference on Service-Oriented and Cloud Computing, pages 49-56. Springer.

Pahl, M.-O. and Aubet, F.-X. (2018). All eyes on you: Distributed multi-dimensional iot microservice anomaly detection. In 201814 th International Conference on Network and Service Management (CNSM), pages 72-80. IEEE.

Pahl, M.-O., Aubet, F.-X., and Liebald, S. (2018). Graph-based iot microservice security. In NOMS 2018-2018 IEEE/IFIP Network Operations and Management Symposium, pages 1-3. IEEE.

Pahl, M.-O. and Donini, L. (2018). Securing iot microservices with certificates. In NOMS 2018-2018 IEEE/IFIP Network Operations and Management Symposium, pages 1-5. IEEE.

Paladi, N., Michalas, A., and Dang, H.-V. (2018). Towards secure cloud orchestration for multi-cloud deployments. In Proceedings of the 5th Workshop on CrossCloud Infrastructures 83 Platforms, pages 1-6.

Panduman, Y. Y. F., Sukaridhoto, S., and Tjahjono, A. (2019). A survey of iot platform comparison for building cyber-physical system architecture. In 2019 International Seminar on Research of Information Technology and Intelligent Systems (ISRITI), pages 238-243. IEEE.

Park, E. and Jeon, K. (2020). Secure volume hot-plugging for containers (industry track). In Proceedings of the 1st International Middleware Conference Industrial Track, pages 38-44.

Paschke, A. (2016). Provalets: Component-based mobile agents as microservices for rule-based data access, processing and analytics. Business $\mathcal{E}$ Information Systems Engineering, 58(5):329340 .

Perrone, G. and Romano, S. P. (2017). The docker security playground: A hands-on approach to the study of network security. In 2017 Principles, Systems and Applications of IP Telecommunications (IPTComm), pages 1-8. IEEE.

Petrovska, J., Memeti, A., and Imeri, F. (2019). Soa approach-identity and access management for the risk management platform. In 2019 8th Mediterranean Conference on Embedded Computing (MECO), pages 1-4. IEEE.

Plaza, A. M., Díaz, J., and Pérez, J. (2018). Software architectures for health care cyberphysical systems: A systematic literature review. Journal of Software: Evolution and Process, 30(7):e1930.

Prandi, C., Melis, A., Prandini, M., Delnevo, G., Monti, L., Mirri, S., and Salomoni, P. (2019). Gamifying cultural experiences across the urban environment. Multimedia Tools and Applications, 78(3):3341-3364.

Preuveneers, D. and Joosen, W. (2017). Access control with delegated authorization policy evaluation for data-driven microservice workflows. Future Internet, 9(4):58. 
Preuveneers, D. and Joosen, W. (2019). Towards multi-party policy-based access control in federations of cloud and edge microservices. In 2019 IEEE European Symposium on Security and Privacy Workshops (EuroS\&PW), pages 29-38. IEEE.

Puliafito, C., Mingozzi, E., Longo, F., Puliafito, A., and Rana, O. (2019). Fog computing for the internet of things: A survey. ACM Transactions on Internet Technology (TOIT), 19(2):1-41.

Pustchi, N., Krishnan, R., and Sandhu, R. (2015). Authorization federation in iaas multi cloud. In Proceedings of the 3rd International Workshop on Security in Cloud Computing, pages 63-71.

Ranawaka, I., Marru, S., Graham, J., Bisht, A., Basney, J., Fleury, T., Gaynor, J., Wannipurage, D., Christie, M., Mahmoud, A., et al. (2020). Custos: Security middleware for science gateways. In Practice and Experience in Advanced Research Computing, pages 278-284.

Ranjbar, A., Komu, M., Salmela, P., and Aura, T. (2017). Synaptic: Secure and persistent connectivity for containers. In 2017 17th IEEE/ACM International Symposium on Cluster, Cloud and Grid Computing (CCGRID), pages 262-267. IEEE.

Rao, T. R., Mitra, P., Bhatt, R., and Goswami, A. (2018). The big data system, components, tools, and technologies: a survey. Knowledge and Information Systems, pages 1-81.

Ravichandran, A., Taylor, K., and Waterhouse, P. (2016). DevOps for Digital Leaders. CA.

Razian, M., Fathian, M., and Buyya, R. (2020). Arc: Anomaly-aware robust cloud-integrated iot service composition based on uncertainty in advertised quality of service values. Journal of Systems and Software, 164:110557.

Razzaq, A. (2020). A systematic review on software architectures for iot systems and future direction to the adoption of microservices architecture. SN Computer Science, 1(6):1-30.

Redelinghuys, A., Basson, A., and Kruger, K. (2019). A six-layer architecture for the digital twin: a manufacturing case study implementation. Journal of Intelligent Manufacturing, pages $1-20$.

Reed, J. P. (2020). Beyond the'fix-it'treadmill. Communications of the ACM, 63(5):58-63.

Reyna, A., Martín, C., Chen, J., Soler, E., and Díaz, M. (2018). On blockchain and its integration with iot. challenges and opportunities. Future generation computer systems, 88:173-190.

Roca, S., Sancho, J., García, J., and Alesanco, Á. (2020). Microservice chatbot architecture for chronic patient support. Journal of biomedical informatics, 102:103305.

Ruan, H., Chen, B., Peng, X., and Zhao, W. (2019). Deeplink: Recovering issue-commit links based on deep learning. Journal of Systems and Software, 158:110406.

Russinovich, M., Costa, M., Fournet, C., Chisnall, D., Delignat-Lavaud, A., Clebsch, S., Vaswani, K., and Bhatia, V. (2021). Toward confidential cloud computing: Extending hardware-enforced cryptographic protection to data while in use. Queue, 19(1):49-76.

Safaryan, O., Pinevich, E., Roshchina, E., Cherckesova, L., and Kolennikova, N. (2020). Information system development for restricting access to software tool built on microservice architecture. In E3S Web of Conferences, volume 224. EDP Sciences.

Salibindla, J. (2018). Microservices api security. International Journal of Engineering Research $\&$ Technology, 7(1):277-281.

Salomoni, D., Campos, I., Gaido, L., de Lucas, J. M., Solagna, P., Gomes, J., Matyska, L., Fuhrman, P., Hardt, M., Donvito, G., et al. (2018). Indigo-datacloud: a platform to facilitate seamless access to e-infrastructures. Journal of Grid Computing, 16(3):381-408.

Schlossnagle, T. (2017). Monitoring in a devops world. Queue, 15(6):35-45. 
Schlossnagle, T. (2018). Monitoring in a devops world. Communications of the ACM, 61(3):58-61.

Shahin, M., Zahedi, M., Babar, M. A., and Zhu, L. (2019). An empirical study of architecting for continuous delivery and deployment. Empirical Software Engineering, 24(3):1061-1108.

Sharma, P., Lawrenz, S., and Rausch, A. (2020). Towards trustworthy and independent data marketplaces. In Proceedings of the 2020 The 2nd International Conference on Blockchain Technology, pages 39-45.

ShuLin, Y. and JiePing, H. (2020). Research on unified authentication and authorization in microservice architecture. In 2020 IEEE 20th International Conference on Communication Technology (ICCT), pages 1169-1173. IEEE.

Sialm, G. and Knittl, S. (2016). Bring your own identity-case study from the swiss government. In Annual Privacy Forum, pages 38-47. Springer.

Sim, A. X. A., Barus, O. P., and Jaya, F. (2019). Lessons learned in applying reactive system in microservices. In Journal of Physics: Conference Series, volume 1175, page 012101. IOP Publishing.

Soldani, J., Tamburri, D. A., and Van Den Heuvel, W.-J. (2018). The pains and gains of microservices: A systematic grey literature review. Journal of Systems and Software, 146:215232 .

Souppaya, M., Morello, J., and Scarfone, K. (2017). Application container security guide (2nd draft). Technical report, National Institute of Standards and Technology.

Stallenberg, D. M. and Panichella, A. (2019). Jcomix: a search-based tool to detect xml injection vulnerabilities in web applications. In Proceedings of the 2019 27th ACM Joint Meeting on European Software Engineering Conference and Symposium on the Foundations of Software Engineering, pages 1090-1094.

Stock, D., Schel, D., and Bauernhansl, T. (2020). Middleware-based cyber-physical production system modeling for operators. Procedia Manufacturing, 42:111-118.

Stocker, M., Zimmermann, O., Zdun, U., Lübke, D., and Pautasso, C. (2018). Interface quality patterns: Communicating and improving the quality of microservices apis. In Proceedings of the 23rd European Conference on Pattern Languages of Programs, pages 1-16.

Sultan, S., Ahmad, I., and Dimitriou, T. (2019). Container security: Issues, challenges, and the road ahead. IEEE Access, 7:52976-52996.

Sun, Y., Nanda, S., and Jaeger, T. (2015). Security-as-a-service for microservices-based cloud applications. In 2015 IEEE 7th International Conference on Cloud Computing Technology and Science (CloudCom), pages 50-57. IEEE.

Sundelin, A., Gonzalez-Huerta, J., and Wnuk, K. (2020). The hidden cost of backward compatibility: when deprecation turns into technical debt-an experience report. In Proceedings of the 3rd International Conference on Technical Debt, pages 67-76.

Suneja, S., Kanso, A., and Isci, C. (2019). Can container fusion be securely achieved? In Proceedings of the 5th International Workshop on Container Technologies and Container Clouds, pages 31-36.

Surantha, N. and Ivan, F. (2019). Secure kubernetes networking design based on zero trust model: A case study of financial service enterprise in indonesia. In International Conference on Innovative Mobile and Internet Services in Ubiquitous Computing, pages 348-361. Springer.

Syed, M. H. and Fernandez, E. B. (2017). The container manager pattern. In Proceedings of the 22nd European Conference on Pattern Languages of Programs, pages 1-9. 
Syed, M. H. and Fernandez, E. B. (2018). A reference architecture for the container ecosystem. In Proceedings of the 13th International Conference on Availability, Reliability and Security, pages 1-6.

Taha, M. B., Talhi, C., and Ould-Slimanec, H. (2019). A cluster of cp-abe microservices for vanet. Procedia Computer Science, 155:441 - 448. The 16th International Conference on Mobile Systems and Pervasive Computing (MobiSPC 2019),The 14th International Conference on Future Networks and Communications (FNC-2019), The 9th International Conference on Sustainable Energy Information Technology.

Taherizadeh, S. and Grobelnik, M. (2020). Key influencing factors of the kubernetes auto-scaler for computing-intensive microservice-native cloud-based applications. Advances in Engineering Software, 140:102734.

Tchoubraev, D. and Wiczynski, D. (2015). Swiss tso integrated operational planning, optimization and ancillary services system. In 2015 IEEE Eindhoven PowerTech, pages 1-6. IEEE.

Tenev, T. and Tsvetanov, S. (2020). Recommendations for enhancing security in microservice environment altered in an intelligent way. In 2020 International Conference on Software, Telecommunications and Computer Networks (SoftCOM), pages 1-6. IEEE.

Thanh, T. Q., Covaci, S., Magedanz, T., Gouvas, P., and Zafeiropoulos, A. (2016). Embedding security and privacy into the development and operation of cloud applications and services. In 2016 17th International Telecommunications Network Strategy and Planning Symposium (Networks), pages 31-36. IEEE.

Thramboulidis, K., Vachtsevanou, D. C., and Kontou, I. (2019). Cpus-iot: A cyber-physical microservice and iot-based framework for manufacturing assembly systems. Annual Reviews in Control, 47:237-248.

Tien, C.-W., Huang, T.-Y., Tien, C.-W., Huang, T.-C., and Kuo, S.-Y. (2019). Kubanomaly: Anomaly detection for the docker orchestration platform with neural network approaches. Engineering Reports, page e12080.

Torkura, K. A., Sukmana, M. I., Cheng, F., and Meinel, C. (2017a). Leveraging cloud native design patterns for security-as-a-service applications. In 2017 IEEE International Conference on Smart Cloud (SmartCloud), pages 90-97. IEEE.

Torkura, K. A., Sukmana, M. I., and Kayem, A. V. (2018). A cyber risk based moving target defense mechanism for microservice architectures. In 2018 IEEE Intl Conf on Parallel 85 Distributed Processing with Applications, Ubiquitous Computing 83 Communications, Big Data 83 Cloud Computing, Social Computing \& Networking, Sustainable Computing \& Communications (ISPA/IUCC/BDCloud/SocialCom/SustainCom), pages 932-939. IEEE.

Torkura, K. A., Sukmana, M. I., and Meinel, C. (2017b). Integrating continuous security assessments in microservices and cloud native applications. In Proceedings of the10th International Conference on Utility and Cloud Computing, pages 171-180.

Tourani, R., Bos, A., Misra, S., and Esposito, F. (2019). Towards security-as-a-service in multi-access edge. In Proceedings of the 4th ACM/IEEE Symposium on Edge Computing, pages $358-363$.

Trihinas, D., Tryfonos, A., and Dikaiakos, M. D. (2016). Designing scalable and secure microservices by embracing devops-as-a-service offerings.

Trihinas, D., Tryfonos, A., Dikaiakos, M. D., and Pallis, G. (2018). Devops as a service: Pushing the boundaries of microservice adoption. IEEE Internet Computing, 22(3):65-71.

Trnka, M., Černý, T., and Stickney, N. (2018). Survey of authentication and authorization for the internet of things. Security and Communication Networks, 2018:1-17. 
Troiano, E., Soldatos, J., Polyviou, A., Polyviou, A., Mamelli, A., and Drakoulis, D. (2019). Big data platform for integrated cyber and physical security of critical infrastructures for the financial sector: Critical infrastructures as cyber-physical systems. In Proceedings of the 11th International Conference on Management of Digital EcoSystems, pages 262-269.

Trubiani, C., Bran, A., van Hoorn, A., Avritzer, A., and Knoche, H. (2018). Exploiting load testing and profiling for performance antipattern detection. Information and Software Technology, 95:329-345.

Truong, H.-L. and Klein, P. (2020). Devops contract for assuring execution of iot microservices in the edge. Internet of Things, 9:100150.

Tuma, K., Sion, L., Scandariato, R., and Yskout, K. (2020). Automating the early detection of security design flaws. In Proceedings of the 23rd ACM/IEEE International Conference on Model Driven Engineering Languages and Systems, pages 332-342.

Vale, A. P., Marquez, G., and Astudillo (2019). Security mechanisms used in microservices-based systems: A systematic mapping.

Vaquero, L. M., Cuadrado, F., Elkhatib, Y., Bernal-Bernabe, J., Srirama, S. N., and Zhani, M. F. (2019). Research challenges in nextgen service orchestration. Future Generation Computer Systems, 90:20-38.

Varghese, B. and Buyya, R. (2018). Next generation cloud computing: New trends and research directions. Future Generation Computer Systems, 79:849-861.

Vassilakis, V., Panaousis, E., and Mouratidis, H. (2016). Security challenges of small cell as a service in virtualized mobile edge computing environments. In IFIP International Conference on Information Security Theory and Practice, pages 70-84. Springer.

Walker, A. and Cerny, T. (2020). On cloud computing infrastructure for existing code-clone detection algorithms. ACM SIGAPP Applied Computing Review, 20(1):5-14.

Walsh, K. and Manferdelli, J. (2017). Mechanisms for mutual attested microservice communication. In Companion Proceedings of the10th International Conference on Utility and Cloud Computing, pages 59-64.

Wang, L., Zhao, N., Chen, J., Li, P., Zhang, W., and Sui, K. (2020). Root-cause metric location for microservice systems via log anomaly detection. In 2020 IEEE International Conference on Web Services (ICWS), pages 142-150. IEEE.

Wang, P., Xu, J., Ma, M., Lin, W., Pan, D., Wang, Y., and Chen, P. (2018). Cloudranger: root cause identification for cloud native systems. In 2018 18th IEEE/ACM International Symposium on Cluster, Cloud and Grid Computing (CCGRID), pages 492-502. IEEE.

Waseem, M., Liang, P., and Shahin, M. (2020). A systematic mapping study on microservices architecture in devops. Journal of Systems and Software, 170:110798.

Wen, Z., Lin, T., Yang, R., Ji, S., Ranjan, R., Romanovsky, A., Lin, C., and Xu, J. (2019). Ga-par: Dependable microservice orchestration framework for geo-distributed clouds. IEEE Transactions on Parallel and Distributed Systems, 31(1):129-143.

Westerlund, M. and Kratzke, N. (2018). Towards distributed clouds: A review about the evolution of centralized cloud computing, distributed ledger technologies, and a foresight on unifying opportunities and security implications. In 2018 International Conference on High Performance Computing 83 Simulation (HPCS), pages 655-663. IEEE.

Wieber, N. (2020). Automated generation of client-specific backends utilizing existing microservices and architectural knowledge. In 2020 35th IEEE/ACM International Conference on Automated Software Engineering (ASE), pages 1158-1160. IEEE. 
Wu, X., Hou, K., Leng, X., Li, X., Yu, Y., Wu, B., and Chen, Y. (2019). State of the art and research challenges in the security technologies of network function virtualization. IEEE Internet Computing.

$\mathrm{Xu}$, B. and Bian, J. (2020). A cloud robotic application platform design based on the microservices architecture. In 2020 International Conference on Control, Robotics and Intelligent System, pages $13-18$.

Xu, R., Jin, W., and Kim, D. (2019a). Microservice security agent based on api gateway in edge computing. Sensors, 19(22):4905.

Xu, R., Nikouei, S. Y., Chen, Y., Blasch, E., and Aved, A. (2019b). Blendmas: A blockchainenabled decentralized microservices architecture for smart public safety. In 2019 IEEE International Conference on Blockchain (Blockchain), pages 564-571. IEEE.

Yang, X., Wallom, D., Waddington, S., Wang, J., Shaon, A., Matthews, B., Wilson, M., Guo, Y., Guo, L., Blower, J. D., et al. (2014). Cloud computing in e-science: research challenges and opportunities. The Journal of Supercomputing, 70(1):408-464.

Yang, Y., Zu, Q., Liu, P., Ouyang, D., and Li, X. (2018). Microshare: privacy-preserved medical resource sharing through microservice architecture. International journal of biological sciences, 14(8):907.

Yarygina, T. (2018). Exploring microservice security.

Yarygina, T. and Bagge, A. H. (2018). Overcoming security challenges in microservice architectures. In 2018 IEEE Symposium on Service-Oriented System Engineering (SOSE), pages 11-20. IEEE.

Yarygina, T. and Otterstad, C. (2018). A game of microservices: Automated intrusion response. In IFIP International Conference on Distributed Applications and Interoperable Systems, pages 169-177. Springer.

Yousefpour, A., Fung, C., Nguyen, T., Kadiyala, K., Jalali, F., Niakanlahiji, A., Kong, J., and Jue, J. P. (2019). All one needs to know about fog computing and related edge computing paradigms: A complete survey. Journal of Systems Architecture.

Yu, D., Jin, Y., Zhang, Y., and Zheng, X. (2019). A survey on security issues in services communication of microservices-enabled fog applications. Concurrency and Computation: Practice and Experience, 31(22):e4436.

Yuan, M., Fang, Y., Lv, J., Zheng, S., and Zhou, Z. (2019). Research on power trading platform based on big data and artificial intelligence technology. In IOP Conference Series: Materials Science and Engineering, volume 486, page 012109. IOP Publishing.

Zaheer, Z., Chang, H., Mukherjee, S., and Van der Merwe, J. (2019). eztrust: Networkindependent zero-trust perimeterization for microservices. In Proceedings of the 2019 ACM Symposium on SDN Research, pages 49-61.

Zdun, U., Wittern, E., and Leitner, P. (2019). Emerging trends, challenges, and experiences in devops and microservice apis. IEEE Software, 37(1):87-91.

Zhang, C., Liu, X., Zheng, X., Li, R., and Liu, H. (2020). Fenghuolun: A federated learning based edge computing platform for cyber-physical systems. In 2020 IEEE International Conference on Pervasive Computing and Communications Workshops (PerCom Workshops), pages 1-4. IEEE.

Zhang, N., Li, H., Hu, H., and Park, Y. (2017). Towards effective virtualization of intrusion detection systems. In Proceedings of the ACM International Workshop on Security in Software Defined Networks \& Network Function Virtualization, pages 47-50. 
1804

1805

1806

1807

1808

1809

1810

1811

1812

1813

1814

Zhiyi, L., Shahidehpour, M., and Xuan, L. (2018). Cyber-secure decentralized energy management for iot-enabled active distribution networks. Journal of Modern Power Systems and Clean Energy, 6(5):900-917.

Zimmermann, O. (2017a). Architectural refactoring for the cloud: a decision-centric view on cloud migration. Computing, 99(2):129-145.

Zimmermann, O. (2017b). Microservices tenets. Computer Science-Research and Development, $32(3-4): 301-310$.

Zuo, Y., Wu, Y., Min, G., Huang, C., and Pei, K. (2020). An intelligent anomaly detection scheme for micro-services architectures with temporal and spatial data analysis. IEEE Transactions on Cognitive Communications and Networking, 6(2):548-561.

\section{APPENDIX}




\section{Dataset and Research Questions in tabular form}

We partition the dataset into four tables, each representing the categorisation described in Section 5.2.1-i) Theoretical, ii) Applicative, and iii) Theoretical and Applicative publications and iv) Survey. For each table we have 5 columns. The first 4 columns from the left (after the column containing the reference ("Ref.") to the publication from the publications dataset) and grouped under the column group "Group" report the 4 Research Questions Groups as defined in Section 4. The value shown indicates the amount of questions of each group the publications answered. The last column labeled "Q.Num." presents the number of questions having a positive answer. 


\begin{tabular}{|c|c|c|c|c|c|}
\hline \multicolumn{6}{|c|}{ Survey Publications } \\
\hline Ref. & \multicolumn{4}{|c|}{ Group } & Q. Num. \\
\hline Sultan et al. (2019) & 3 & 2 & 2 & 1 & $2,4,5,8,11,13,14,16$ \\
\hline Cerny and Donahoo (2016) & 0 & 0 & 1 & 0 & 13 \\
\hline Westerlund and Kratzke (2018) & 0 & 1 & 1 & 1 & $11,15,16$ \\
\hline Bandeira et al. (2019) & 0 & 0 & 2 & 0 & 13,14 \\
\hline Ahmed et al. (2019) & 0 & 1 & 1 & 0 & 8,13 \\
\hline Di Salle et al. (2016) & 0 & 0 & 1 & 1 & 13,16 \\
\hline Bélair et al. (2019) & 0 & 0 & 2 & 0 & 13,14 \\
\hline Márquez and Astudillo (2019) & 2 & 0 & 2 & 0 & $2,4,13,14$ \\
\hline Puliafito et al. (2019) & 1 & 0 & 0 & 0 & 2 \\
\hline Manu et al. (2016) & 0 & 2 & 1 & 1 & $8,11,13,17$ \\
\hline Lysne et al. (2016) & 2 & 0 & 1 & 0 & $3,4,13$ \\
\hline Panduman et al. (2019) & 1 & 0 & 0 & 0 & 2 \\
\hline Casale et al. (2016) & 0 & 0 & 1 & 1 & 13,16 \\
\hline Soldani et al. (2018) & 1 & 1 & 2 & 3 & $4,8,13,14,16-18$ \\
\hline Almeida et al. (2017) & 1 & 0 & 1 & 0 & 2,13 \\
\hline Yousefpour et al. (2019) & 0 & 1 & 1 & 0 & 11,13 \\
\hline Trnka et al. (2018) & 0 & 0 & 1 & 0 & 13 \\
\hline Adedugbe et al. (2019) & 1 & 1 & 2 & 0 & $3,8,13,14$ \\
\hline Lichtenthäler et al. (2019) & 0 & 1 & 1 & 0 & 11,13 \\
\hline Mohsin and Janjua (2018) & 0 & 1 & 1 & 1 & $11,13,17$ \\
\hline Noura et al. (2019) & 0 & 0 & 1 & 0 & 13 \\
\hline Rao et al. (2018) & 0 & 1 & 1 & 0 & 11,13 \\
\hline Yang et al. (2014) & 1 & 1 & 1 & 0 & $3,11,13$ \\
\hline Yu et al. (2019) & 1 & 1 & 2 & 1 & $2,8,13,14,16$ \\
\hline Casalicchio and lannucci (2020) & 2 & 1 & 1 & 0 & $2,5,11,13$ \\
\hline Plaza et al. (2018) & 0 & 0 & 0 & 1 & 17 \\
\hline Di Francesco et al. (2017) & 2 & 0 & 0 & 2 & $4,5,16,17$ \\
\hline Islam et al. (2016) & 3 & 1 & 0 & 0 & $2,4,5,8$ \\
\hline Vale et al. (2019) & 2 & 2 & 0 & 0 & $2,5,9,11$ \\
\hline Bélair et al. (2019) & 0 & 0 & 2 & 0 & 13,14 \\
\hline Márquez and Astudillo (2019) & 2 & 0 & 2 & 0 & $2,4,13,14$ \\
\hline Puliafito et al. (2019) & 1 & 0 & 0 & 0 & 2 \\
\hline Manu et al. (2016) & 0 & 2 & 1 & 1 & $8,11,13,17$ \\
\hline Lysne et al. (2016) & 2 & 0 & 1 & 0 & $3,4,13$ \\
\hline Panduman et al. (2019) & 1 & 0 & 0 & 0 & 2 \\
\hline Casale et al. (2016) & 0 & 0 & 1 & 1 & 13,16 \\
\hline Soldani et al. (2018) & 1 & 1 & 2 & 3 & $4,8,13,14,16,17,18$ \\
\hline Almeida et al. (2017) & 1 & 0 & 1 & 0 & 2,13 \\
\hline Yousefpour et al. (2019) & 0 & 1 & 1 & 0 & 11,13 \\
\hline Sultan et al. (2019) & 3 & 2 & 2 & 1 & $2,4,5,8,11,13,14,16$ \\
\hline Ahmed et al. (2019) & 0 & 1 & 1 & 0 & 8,13 \\
\hline Trnka et al. (2018) & 0 & 0 & 1 & 0 & 13 \\
\hline Cerny and Donahoo (2016) & 0 & 0 & 1 & 0 & 13 \\
\hline Ahmadvand et al. (2018) & 2 & 7 & 3 & 3 & $2,3,6-18$ \\
\hline Adedugbe et al. (2019) & 1 & 1 & 2 & 0 & $3,8,13,14$ \\
\hline Lichtenthäler et al. (2019) & 0 & 1 & 1 & 0 & 11,13 \\
\hline Mohsin and Janjua (2018) & 0 & 1 & 1 & 1 & $11,13,17$ \\
\hline Niazi et al. (2018) & 0 & 0 & 0 & 0 & \\
\hline Noura et al. (2019) & 0 & 0 & 1 & 0 & 13 \\
\hline Rao et al. (2018) & 0 & 1 & 1 & 0 & 11,13 \\
\hline Yang et al. (2014) & 1 & 1 & 1 & 0 & $3,11,13$ \\
\hline Yu et al. (2019) & 1 & 1 & 2 & 1 & $2,8,13,14,16$ \\
\hline Casalicchio and lannucci (2020) & 2 & 1 & 1 & 0 & $2,5,11,13$ \\
\hline Plaza et al. (2018) & 0 & 0 & 0 & 1 & 17 \\
\hline Di Francesco et al. (2017) & 2 & 0 & 0 & 2 & $4,5,16,17$ \\
\hline Westerlund and Kratzke (2018) & 0 & 1 & 1 & 1 & $11,15,16$ \\
\hline Islam et al. (2016) & 3 & 1 & 0 & 0 & $2,4,5,8$ \\
\hline Vale et al. (2019) & 2 & 2 & 0 & 0 & $2,5,9,11$ \\
\hline Lie et al. (2020) & 2 & 1 & 0 & 3 & $3,4,11,16,17,20$ \\
\hline Ali et al. (2020) & 4 & 0 & 0 & 0 & $2,3,4,5$ \\
\hline de Sousa et al. (2020) & 2 & 1 & 1 & 2 & $2,3,11,13,16,19$ \\
\hline Adam et al. (2020) & 2 & 0 & 0 & 0 & 2,5 \\
\hline Delicato et al. (2020) & 3 & 0 & 0 & 0 & $2,4,5$ \\
\hline Mohamed et al. (2020) & 2 & 0 & 0 & 1 & $2,4,18$ \\
\hline Waseem et al. (2020) & 2 & 1 & 2 & 4 & $2,4,11,13,14,16-19$ \\
\hline Mishra and Otaiwi (2020) & 1 & 1 & 1 & 2 & $2,11,13,16,17$ \\
\hline Niknejad et al. (2020) & 1 & 1 & 0 & 0 & 2,11 \\
\hline Moura and Hutchison (2020) & 2 & 0 & 0 & 0 & 2,4 \\
\hline de Araujo Zanella et al. (2020) & 4 & 2 & 0 & 0 & $2,3,4,5,6,7$ \\
\hline Mohamed et al. (2020) & 2 & 0 & 0 & 1 & $2,4,18$ \\
\hline Razzaq (2020) & 2 & 1 & 3 & 4 & $2,3,11,13-19$ \\
\hline Wu et al. (2019) & 1 & 2 & 0 & 0 & $2,6,11$ \\
\hline
\end{tabular}

\begin{tabular}{|c|c|c|c|c|c|}
\hline \multicolumn{6}{|c|}{ Applicative Publications } \\
\hline 年 & \multicolumn{4}{|c|}{ Group } & Q. Num. \\
\hline George and Mahmoud (2017) & $\frac{3}{2}$ & 0 & 1 & 0 & $2,5,13$ \\
\hline Thramboulidis et al. (2019) & 1 & 1 & 1 & 0 & $5,8,13$ \\
\hline Ciavotta et al. (2017) & 0 & 1 & 1 & 1 & $11,13,17$ \\
\hline Morris (2017) & 2 & 2 & 1 & 0 & $3,5,8,11,13$ \\
\hline Fetzer et al. (2017) & 2 & 3 & 2 & 1 & $2,3,6-8,13,14,16$ \\
\hline Jita and Pieterse (2018) & 1 & 0 & 2 & 0 & $2,13,14$ \\
\hline Perrone and Romano (2017) & 0 & 0 & 1 & 1 & 13,17 \\
\hline Pahl and Aubet (2018) & 1 & 0 & 2 & 0 & $3,13,14$ \\
\hline Sialm and Knittl (2016) & 0 & 0 & 1 & 0 & 13 \\
\hline Du et al. (2018) & 1 & 1 & 1 & 0 & $3,8,13$ \\
\hline Kalske et al. (2017) & 1 & 1 & 2 & 1 & $2,8,13,14,16$ \\
\hline Nehme et al. (2018) & 1 & 1 & 1 & 0 & $2,8,13$ \\
\hline Nikoloudakis et al. (2019) & 0 & 2 & 1 & 0 & $8,11,13$ \\
\hline Salomoni et al. (2018) & 0 & 0 & 2 & 1 & $13,14,16$ \\
\hline Stallenberg and Panichella (2019) & 3 & 1 & 0 & 0 & $2,3,5,7$ \\
\hline Morris (2017) & 2 & 2 & 1 & 0 & $3,5,8,11,13$ \\
\hline Fetzer et al. (2017) & 2 & 3 & 2 & 1 & $2,3,6,7,8,13,14,16$ \\
\hline Jita and Pieterse (2018) & 1 & 0 & 2 & 0 & $2,13,14$ \\
\hline Perrone and Romano (2017) & 0 & 0 & 1 & 1 & 13,17 \\
\hline Pahl and Aubet (2018) & 1 & 0 & 2 & 0 & $3,13,14$ \\
\hline Sialm and Knittl (2016) & 0 & 0 & 1 & 0 & 13 \\
\hline Cerny and Donahoo (2016) & 0 & 0 & 1 & 0 & 13 \\
\hline Du et al. (2018) & 1 & 1 & 1 & 0 & $3,8,13$ \\
\hline Kalske et al. (2017) & 1 & 1 & 2 & 1 & $2,8,13,14,16$ \\
\hline Nehme et al. (2018) & 1 & 1 & 1 & 0 & $2,8,13$ \\
\hline Nikoloudakis et al. (2019) & 0 & 2 & 1 & 0 & $8,11,13$ \\
\hline Salomoni et al. (2018) & 0 & 0 & 2 & 1 & $13,14,16$ \\
\hline Stallenberg and Panichella (2019) & 3 & 1 & 0 & 0 & $2,3,5,7$ \\
\hline Park and Jeon (2020) & 1 & 1 & 1 & 0 & $2,11,13$ \\
\hline $\mathrm{Xu}$ and Bian (2020) & 0 & 1 & 1 & 1 & $11,13,16$ \\
\hline Brondolin and Santambrogio (2020) & 1 & 2 & 1 & 0 & $2,6,11,13$ \\
\hline Ma et al. (2020) & 1 & 1 & 0 & 1 & $3,7,16$ \\
\hline Olsthoorn et al. (2020) & 1 & 1 & 0 & 1 & $3,7,16$ \\
\hline Chen et al. (2020) & 2 & 1 & 0 & 0 & $2,3,11$ \\
\hline Zuo et al. (2020) & 2 & 1 & 1 & 0 & $2,3,11,13$ \\
\hline Luntovskyy and Shubyn (2020) & 1 & 3 & 1 & 2 & $2,6,7,11,13,16,19$ \\
\hline Ghuge et al. (2020) & 3 & 1 & 0 & 0 & $2,3,4,11$ \\
\hline Gerostathopoulos et al. (2020) & 1 & 1 & 0 & 0 & 2,11 \\
\hline Zhang et al. (2020) & 1 & 1 & 0 & 0 & 2,11 \\
\hline Hang et al. (2020) & 3 & 1 & 0 & 0 & $2,3,4,11$ \\
\hline Forti et al. (2020) & 3 & 3 & 1 & 0 & $2,3,4,6,7,11,13$ \\
\hline Stock et al. (2020) & 2 & 0 & 0 & 1 & $2,4,18$ \\
\hline Hasan and Starly (2020) & 1 & 0 & 1 & 0 & 2,13 \\
\hline Kallergis et al. (2020) & 2 & 1 & 1 & 0 & $2,4,11,13$ \\
\hline Amir-Mohammadian and Kari (2020) & 2 & 1 & 0 & 0 & $2,4,11$ \\
\hline Roca et al. (2020) & 3 & 1 & 1 & 0 & $2,3,4,11,13$ \\
\hline Bromberg and Gitzinger (2020) & 2 & 4 & 1 & 2 & $2,3,6-8,11,13,16,18$ \\
\hline Jaworski et al. (2019) & 2 & 1 & 1 & 0 & $2,4,11,13$ \\
\hline
\end{tabular}

\begin{tabular}{|c|c|c|c|c|l|}
\hline Ref. & \multicolumn{5}{|c|}{ Group } \\
\hline G1 & G2 & G3 & G4 & Q. Num. \\
\hline ShuLin and JiePing (2020) & 3 & 1 & 1 & 0 & $2,3,4,11,13$ \\
Dilshan et al. (2020) & 4 & 1 & 1 & 0 & $2,3,4,5,11,13$ \\
Flora (2020) & 3 & 3 & 1 & 0 & $2,3,4,6,7,11,13$ \\
Flora et al. (2020) & 3 & 3 & 0 & 0 & $2,3,4,6,7,11$ \\
Bogatinovski et al. (2020) & 2 & 0 & 0 & 0 & 2,4 \\
Damis et al. (2020) & 3 & 1 & 0 & 0 & $2,3,4,11$ \\
Iraqi and El Bakkali (2020) & 3 & 1 & 1 & 0 & $2,3,4,11,13$ \\
Dewanta (2020) & 3 & 1 & 1 & 0 & $2,3,4,11,13$ \\
Bumblauskas et al. (2020) & 1 & 0 & 0 & 0 & 2 \\
Giaimo et al. (2020) & 2 & 0 & 0 & 2 & $2,4,16,17$ \\
Lenarduzzi et al. (2020) & 2 & 1 & 1 & 2 & $2,4,11,13,16,17$ \\
Mann (2020) & 3 & 1 & 0 & 1 & $2,3,4,9,16$ \\
Costa et al. (2020) & 0 & 1 & 1 & 3 & $11,13,16,17,18$ \\
Fahmideh and Zowghi (2020) & 1 & 0 & 0 & 2 & $2,18,19$ \\
Razian et al. (2020) & 1 & 0 & 0 & 0 & 2 \\
Taherizadeh and Grobelnik (2020) & 0 & 1 & 1 & 0 & 11,13 \\
Safaryan et al. (2020) & 2 & 1 & 0 & 0 & $2,4,11$ \\
de Toledo et al. (2020) & 0 & 1 & 1 & 1 & $11,13,16$ \\
Alulema et al. (2020) & 0 & 1 & 1 & 3 & $11,13,16,17,19$ \\
Kapferer and Zimmermann (2020) & 0 & 0 & 1 & 2 & $13,16,19$ \\
Redelinghuys et al. (2019) & 2 & 1 & 0 & 0 & $2,3,11$ \\
Dash et al. (2020) & 2 & 1 & 0 & 0 & $2,3,11$ \\
Kwon et al. (2020) & 3 & 1 & 0 & 0 & $2,3,4,11$ \\
Khan and Shameem (2020) & 1 & 1 & 1 & 3 & $2,11,13,16,17,18$ \\
DesLauriers et al. (2020) & 1 & 1 & 1 & 2 & $2,11,13,16,17$ \\
Bertolino et al. (2020) & 0 & 1 & 1 & 1 & $11,13,16$ \\
Di Sanzo et al. (2021) & 2 & 1 & 0 & 1 & $2,4,11,16$ \\
Moreira et al. (2020) & 2 & 1 & 1 & 2 & $2,4,11,13,16,17$ \\
Li et al. (2019) & 2 & 1 & 1 & 0 & $2,3,11,13$ \\
& & & & & \\
& & & & & \\
& & & &
\end{tabular}


Theoretical Publications (2/3)

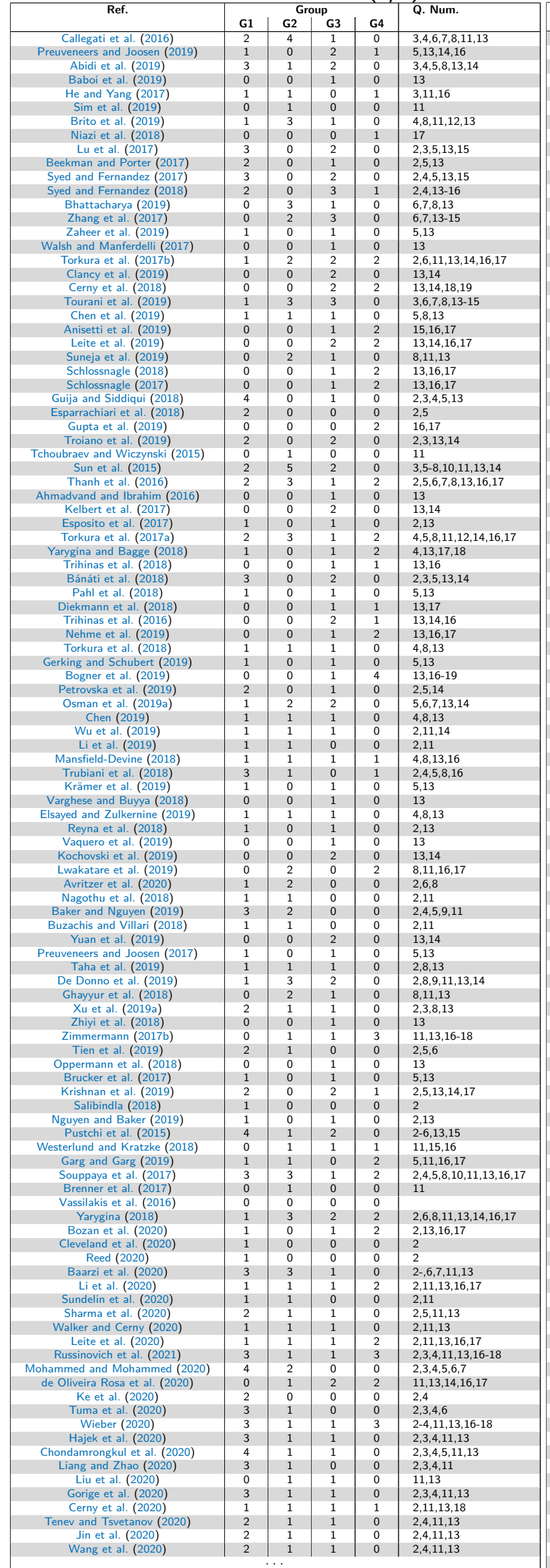

Theoretical Publications (3/3)

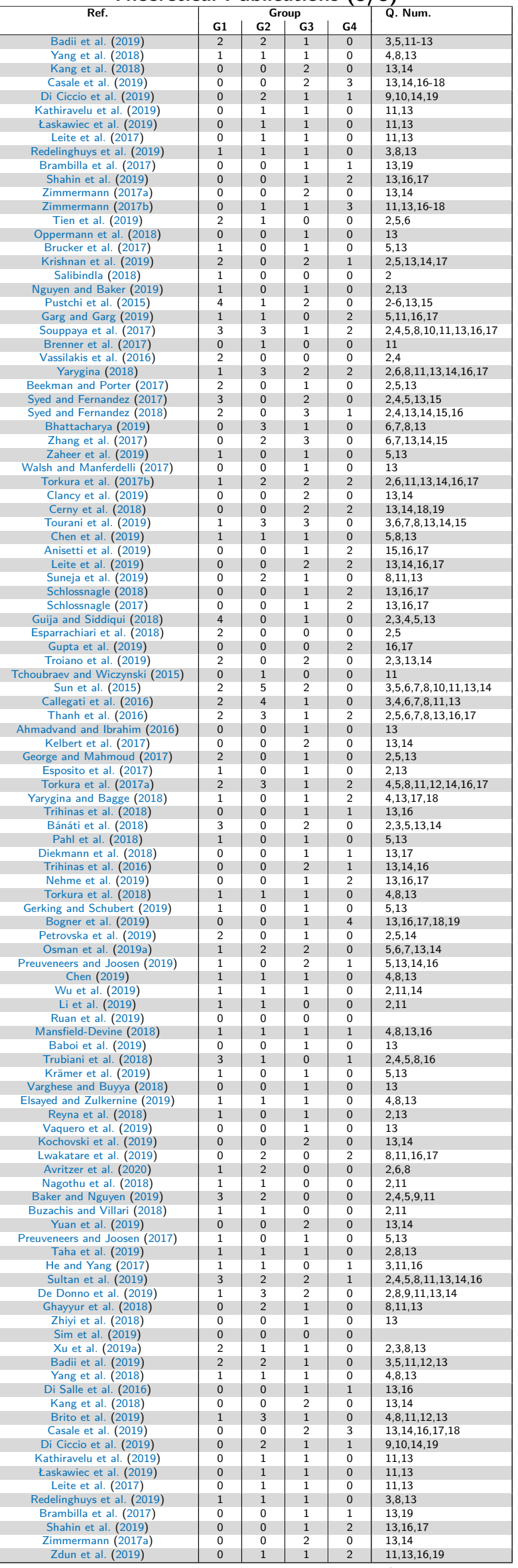


Theoretical and Applicative Publications

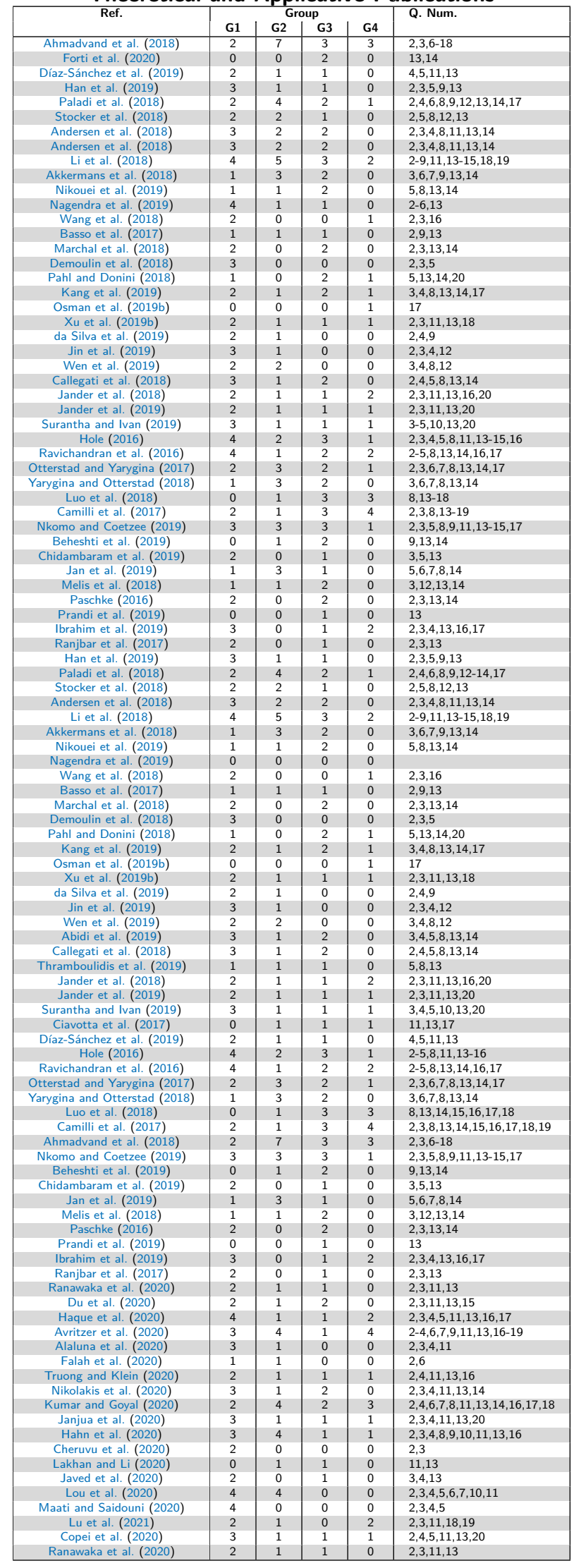

